\title{
ON THE INTERFACE BETWEEN OPTIMAL PERIODIC AND CONTINUOUS DIVIDEND STRATEGIES IN THE PRESENCE OF TRANSACTION COSTS
}

BY

BENJAMIN AVANZI, VinCENT TU AND BERNARD WONG

\begin{abstract}
In the classical optimal dividends problem, dividend decisions are allowed to be made at any point in time - according to a continuous strategy. Depending on the surplus process that is considered and whether dividend payouts are bounded or not, optimal strategies are generally of a band, barrier or threshold type. In reality, while surpluses change continuously, dividends are generally paid on a periodic basis. Because of this, the actuarial literature has recently considered strategies where dividends are only allowed to be distributed at (random) discrete times - according to a periodic strategy.

In this paper, we focus on the Brownian risk model. In this context, the optimal continuous and periodic strategies have previously been shown (independently of one another) to be of barrier type. For the first time, we consider a model where both strategies are used. In such a hybrid strategy, decisions are allowed to be made either at any time (continuously), or periodically at a lower cost. This proves optimal in some cases. We also determine under which combination of parameters a pure continuous, pure periodic or hybrid (including both continuous and periodic dividend payments) barrier strategy is optimal. Interestingly, the hybrid strategy lies in-between periodic and continuous strategies, which provides some interesting insights. Results are illustrated.
\end{abstract}

\section{KEYWORDS}

Brownian motion, Stochastic control, Dividends, Hybrid strategies, Barrier strategies, Transaction costs.

\section{INTRODUCTION}

\subsection{Motivation and literature review}

In actuarial risk theory, stochastic processes are used to model the surplus of a company. In the early 20 th century, the probability of ruin was used as a 
criterion to assess the stability of a company, that is, the probability that the surplus becomes non-positive at some point in future; see, for instance, Bühlmann (1970) or Asmussen and Albrecher (2010). Because more surplus always leads to a lower probability of ruin, the probability of ruin can be seen as a one-sided criterion, suggesting that we should let the surplus grow to infinity. Because of this unrealistic feature, Bruno de Finetti (1957) proposed an alternative formulation that considers the expected present value of dividends (surplus leakages) until ruin as a stability criterion. This is a more balanced criterion in that more dividends lead to an earlier ruin, which itself decreases the expected amount of dividends received later. This proposition led to the study of optimal dividend strategies in many actuarial surplus models, see Avanzi (2009) and Albrecher and Thonhauser (2009) for reviews.

The traditional approach of formulating the optimal dividend problems using de Finetti's criterion is to assume that dividend decisions are made at any point in time, which is here referred to as a continuous strategy. The optimal continuous dividend strategies are studied by many authors in the literature. Gerber (1972) shows that an optimal continuous strategy always exists in both discrete and continuous Markovian surplus processes. Jeanblanc-Picqué and Shiryaev (1995) prove that the continuous barrier strategies are optimal when dividends are subjected to proportional transaction costs in the Brownian risk model and Shreve et al. (1984) show the optimality of the barrier strategies in a general class of diffusion models.

The objective of maximising the expected present value of dividends until ruin has some shortcomings. Gerber (1974) pointed out that the resulting optimal dividend strategies may not be realistic. For instance, dividend payments are often irregular in a barrier strategy, which is arguably an unrealistic feature. Dividend payments tend to occur periodically in reality. This led recently to an alternative formulation of the optimal dividend problems, whereby dividend decisions are assumed to be made on discrete and exogenous time points (see, for example, Albrecher et al., 2011a,b). This will be here referred to as a periodic strategy. However, periodic strategies with deterministic dividend decision times do not preserve the time-Markovian structure and this leads to intractable models. Albrecher et al. (2011a) use an "Erlangisation" approach, which approximates deterministic inter-dividend-decision times with an $\operatorname{Erlang}(n)$ random variable; see also, for example, Avanzi et al. (2013) and Choi and Cheung (2014). In the case of exponential (Erlang(1)) inter-dividend-decision times, Albrecher et al. (2011b) study the periodic barrier strategies by deriving the expected present value of dividends and the associated optimal periodic barrier. The optimality of the periodic barrier strategies are studied by Wei et al. (2012) in the Brownian risk model with regime-switching and Avanzi et al. (2014) in the dual model with diffusion.

In principle, a company that distributes dividends periodically still has access to the surplus at any time. The company may experience significant growth in-between periodic dividend times and may wish to distribute a portion of the growth as dividends immediately. It is plausible to assume that a company 
follows a periodic strategies where dividend decisions arrive periodically. In the meantime, the company also has the opportunity to distribute dividends at any time but possibly at a different (higher) cost. Periodic dividends are typically perceived as an ongoing commitment (e.g., annual dividends) and additional payouts (in various forms) are used to distribute cash due to temporary growth (see, for example, Jagannathan et al., 2000). Indeed, Morningstar (2014) explains

From time to time, companies pay out special dividends when they have had an extraordinarily good period of profitability. These dividends fall outside the scope of the "normal" half-year or full-year result.

This is observed in practice (see, e.g., Woodside Petroleum, 2013; Westfarmers, 2014). Motivated by this, we propose a class of hybrid dividend strategies that allows for a combination of periodic and continuous decision-making. By assuming that dividends distributed from periodic and continuous decisions are subjected to different levels of proportional transaction costs, we find the optimal hybrid strategies that maximise the expected present value of dividends until ruin. Our results show that by varying the ratio between the two transaction costs, the optimal hybrid strategies change from continuous, to hybrid and to periodic barrier strategies. Results are illustrated using numerical examples.

\subsection{Structure of the paper}

In Section 2, we give definitions for stationary, continuous, periodic and hybrid dividend strategies, as well as provide mathematical formulation for the surplus process, expected present value of dividends until ruin and the optimisation problem. In Section 3, we derive closed-form solutions for the expected present value of dividends of hybrid barrier strategies with and without liquidation by solving associated differential equations. We next use the associated verification lemma to show, in Section 4, the optimality of continuous, hybrid and periodic barrier strategies in the set of all hybrid dividend strategies under different combinations of proportional transaction costs. Lastly, we provide some numerical illustrations in Section 5.

\section{MOdel FORMULATION}

\subsection{Definitions of stationary, continuous, periodic and hybrid dividend strategies}

Using stationary strategies has an advantage of reducing the dimensionality of optimisation problems. To quote Bellman (1954), "if at any particular time we know what to do, it is never necessary to know the decisions required at subsequent times". Such strategies are referred to as stationary in Morill (1966) and since then stationarity became a natural (and often implicit) assumption used in the literature. 
From here on, all the strategies defined in the paper are assumed to be stationary. This concept of stationarity is essential to motivate the study in this paper. Note that a stationary strategy will be the same for any time $t \geq 0$. This means that for a given surplus level, the strategy must be applied no matter what. This is where periodic strategies differ from continuous ones, and where they can potentially beat continuous strategies. If the continuous strategies were not stationary, then periodic strategies would be just a subset of them and the calculations in this paper would not make sense; but they are not; see also Avanzi et al. (2014, last paragraph of page 223). Next, we give definitions of continuous and periodic dividend strategies.

Definition 2.1. When a continuous dividend strategy is applied, dividend decisions are made continuously at every instant in time. At any given time $t$, the continuous strategy maps the current value of the surplus $X(t)$ to a particular value of dividend distributed. We denote the set of continuous strategies as $\mathcal{D}_{C}$.

Definition 2.2. When a periodic dividend strategy is applied, dividend decisions only occur at discrete countable time points. We denote these decision times as $\left\{T_{1}, T_{2}, T_{3}, \ldots\right\}$. They are exogenous and independent of the strategy and surplus. If we are at a dividend decision time - if the current time is $t=T_{k}$, a periodic strategy maps the current value of the surplus $X\left(T_{k}\right)$ to a particular value. We denote the set of periodic strategies as $\mathcal{D}_{P}$.

For a periodic dividend strategy to be stationary and for the principles of dynamic programming to be able to be applied (as required in this paper), the exogenous process generating the dividend decision times $\left\{T_{1}, T_{2}, T_{3}, \ldots\right\}$ must be Markovian. In this paper, we assume that inter-dividend-decision times are exponential, but this could be generalised (to $\operatorname{Erlang}(n)$, for instance). So far, continuous and periodic strategies were considered separately. In this paper, we combine them, which leads to the concept of hybrid dividend strategy.

Definition 2.3. When a hybrid dividend strategy is applied, dividend decisions can be made continuously andlor periodically in the spirit of Definitions 2.1 and 2.2, respectively. The set of hybrid dividend strategies is denoted as $\mathcal{D}_{H}$.

The set of hybrid dividend strategies is a bigger family of strategies that contains both $\mathcal{D}_{P}$ and $\mathcal{D}_{C}$. In the remainder of the paper, when dividends can be paid only "continuously" or "periodically" (so when the strategy is not hybrid), then we will qualify those strategies as pure.

\subsection{Model formulation}

Let $(\Omega, \mathcal{F}, \mathrm{P})$ be the probability space on which the surplus process is defined. The surplus process $\{U(t), t \geq 0\}$ follows a Brownian risk model

$$
U(t)=x+\mu t+\sigma W(t)
$$


where $x$ is the initial surplus, $\mu$ is the drift parameter and $\sigma$ represents the volatility. The process $\{W(t), t \geq 0\}$ is a standard Brownian motion. We assume that dividends are distributed from the surplus according to a hybrid strategy (see Definition 2.3) and the surplus process after dividend distribution is defined as

$$
X(t)=x+\mu t+\sigma W(t)-D(t),
$$

where $\{D(t), t \geq 0\}$ represents the aggregate dividends process before transaction costs, with $D(0)=0$. In a hybrid dividend strategy $\Theta_{H}$, the aggregate dividend process before transaction costs comes from two sources, aggregate continuous dividends $\left\{D_{c}(t), t \geq 0\right\}$ and aggregate periodic dividends $\left\{D_{p}(t), t \geq 0\right\}$, i.e.,

$$
D(t)=D_{c}(t)+D_{p}(t) .
$$

We assume that the dividend payments $D_{c}(t)$ are subject to proportional transaction costs and use $\eta_{c} \in[0,1]$ to represent the net proportion of continuous dividends received per dollar. In addition, periodic dividend decisions times $\left\{T_{1}, T_{2}, T_{3} \ldots\right\}$ stem from a Poisson process $\left\{N_{\gamma}(t), t \geq 0\right\}$ with intensity $\gamma>0$, which is independent of the surplus process. We denote a periodic dividend strategy by $\left\{\vartheta_{t}, t \geq 0\right\}$ and a dividend of size $\vartheta_{T_{k}}$ is distributed from the surplus at each periodic dividend decision time $T_{k}$ for $k=1,2,3, \ldots$. So the aggregate periodic dividend process before transaction costs $\left\{D_{p}(t), t \geq 0\right\}$ can be written as

$$
D_{p}(t)=\sum_{k=1}^{\infty} \vartheta_{T_{k}} I_{\left\{T_{k}<t\right\}}=\int_{0}^{t} \vartheta_{s} d N_{\gamma}(s) .
$$

We also assume that proportional transaction costs are applied to periodic dividends and denote $\eta_{p} \in[0,1]$ the net proportion of periodic dividends received per dollar.

In summary, for an arbitrary hybrid dividend strategy $\Theta_{H}$, the expected present value of dividends received by the shareholders until ruin occurs is

$$
J\left(x ; \Theta_{H}\right)=\mathbb{E}^{x}\left[\eta_{c} \int_{0}^{\tau} e^{-\delta s} d D_{c}(s)+\eta_{p} \int_{0}^{\tau} e^{-\delta s} \vartheta_{s} d N_{\gamma}(s)\right],
$$

where $\delta$ represents a suitable force of (discounting) interest, and where

$$
\tau=\inf \{t \geq 0: X(t)=0\} .
$$

is the time of ruin.

Note that we do not make any initial assumption about the relative size of the proportional transaction $\operatorname{costs} \eta_{c}$ and $\eta_{p}$. We will show later that different orderings of $\eta_{c}$ and $\eta_{p}$ lead to different optimal strategies. First, consider the case of $\eta_{c} \geq \eta_{p}$. Every dollar distributed from continuous strategies has an equal or higher net gain than every dollar from periodic strategies. It is reasonable to argue that the optimal hybrid strategies in this case is to only distribute dividends via pure continuous strategies (due to time-value of money). Now, consider the 
case of $\eta_{c}<\eta_{p}$. Dividends distributed from periodic strategies yields a higher net gain than continuous strategies. In this case, the optimal hybrid strategies are not so obvious. In this paper, we show that the ratio between proportional transaction $\operatorname{costs} \eta_{c}$ and $\eta_{p}$ determines the structure of the optimal hybrid strategy. More details are provided in Section 4 and summarised in Table 1 on page 18.

\subsection{Definition of admissible strategies and formulation of the optimisation problem}

In searching for the optimal hybrid strategy that maximises the expected present value of dividends after transaction costs, we consider the set of admissible hybrid strategies, denoted as $\mathcal{D}$. A hybrid strategy $\Theta_{H}$ is considered admissible if its associated aggregate dividend process $\{D(t)\}$ is non-decreasing and $\left\{\mathcal{F}_{t}\right\}$-adapted with càdlàg sample paths and $D(0)=0$. In an admissible hybrid strategy, the set of periodic dividend decision times $\mathcal{T}=\left\{T_{1}, T_{2}, \ldots\right\}$ contains stopping times with respect to $\left\{\mathcal{F}_{t}\right\}$ with $0<T_{1}<T_{2}<\ldots$ a.s. Each periodic dividend payment at time $T_{k}, \vartheta_{T_{k}}$, is measurable with respect to $\left\{\mathcal{F}_{T_{k}}\right\}$ for $k=1,2,3, \ldots$. All dividend payments cannot exceed the current surplus level but are allowed to bring the surplus to zero and cause immediate ruin. Such a payment corresponds to a liquidation strategy. More formally,

$$
D(t)-D(t-) \leq X(t-) \text { for all } t \geq 0 .
$$

We denote the expected present value of dividends under the optimal hybrid strategy as

$$
V(x)=\max _{\Theta_{H} \in \mathcal{D}} J\left(x ; \Theta_{H}\right)=\mathbb{E}^{x}\left[\eta_{c} \int_{0}^{\tau} e^{-\delta s} d D_{c}^{*}(s)+\eta_{p} \int_{0}^{\tau} e^{-\delta s} \vartheta_{s}^{*} d N_{\gamma}(s)\right],
$$

where $\left\{D_{c}^{*}(t)\right\}$ is the aggregate dividend process for the optimal continuous strategy and $\left\{\vartheta_{t}^{*}\right\}$ is the payment process associated with the optimal periodic strategy.

\subsection{Further terminology and review of the relevant existing literature}

Before dwelving into further detail, we would like to make some of the terminology clear, and review the most relevant results, which we will need, but have already been established in the literature. Throughout the paper, we will consider four types of barriers:

Barriers within a hybrid barrier strategy: There are two:

- the hybrid continuous barrier $\left(b_{c}\right)$, and

- the hybrid periodic barrier $\left(b_{p}\right)$. 
These operate at the same time. It makes sense to assume that $b_{p}<b_{c}$, otherwise $b_{p}$ will never be reached, and the strategy would then reduce to the pure continuous barrier as defined immediately below.

Barriers within a pure strategy: We refer here to barriers of continuous or periodic type, which will be exclusively used for dividend distribution purposes (not in conjunction with the other). There are also two:

- the pure continuous barrier $\left({ }_{0} b_{c}\right)$, and

- the pure periodic barrier $\left({ }_{0} b_{p}\right)$.

The optimality of the pure continuous and pure periodic barrier strategies has already been established in the current literature; see Gerber (1972) and Avanzi et al. (2014), respectively. The optimal barrier level will be denoted with a star in the superscript. For example, ${ }_{0} b_{c}^{*}$ is the optimal barrier in a pure continuous dividend strategy. We now review the form of the associated expected present value of dividends, which we will need later.

The expected present value of dividends of a pure continuous barrier strategy with proportional transaction costs $\left(1-\eta_{c}\right)$ in the Brownian risk model is denoted as $G_{C}\left(x ;{ }_{0} b_{c}^{*}\right)$, where ${ }_{0} b_{c}^{*}$ is the optimal pure continuous barrier. The function $G_{C}\left(x ;{ }_{0} b_{c}^{*}\right)$ takes the form of

$$
G_{C}\left(x ;{ }_{0} b_{c}^{*}\right)= \begin{cases}\eta_{c} \frac{e^{r_{0} x}-e^{s_{0} x}}{r_{0} e^{r_{0}\left({ }_{0} b_{c}^{*}\right)}-s_{0} e^{s_{0}\left(b_{c}^{*}\right)}}, & x \in\left[0,{ }_{0} b_{c}^{*}\right), \\ \eta_{c}\left(x-{ }_{0} b_{c}\right)+G_{C}\left({ }_{0} b_{c}^{*} ;{ }_{0} b_{c}^{*}\right), & x \in\left[0 b_{c}^{*}, \infty\right),\end{cases}
$$

where the optimal continuous barrier ${ }_{0} b_{c}^{*}$ has the form

$$
{ }_{0} b_{c}^{*}=\frac{1}{r_{0}-s_{0}} \log \left(\frac{s_{0}^{2}}{r_{0}^{2}}\right),
$$

and where the roots $r_{0}$ and $s_{0}$ are defined around (3.12) below; see JeanblancPicqué and Shiryaev (1995).

Consider now the expected present value of the expected present value of dividends of a pure periodic barrier strategy with transaction costs $\left(1-\eta_{p}\right)$, denoted by $G_{P}\left(x ;{ }_{0} b_{p}^{*}\right)$, where ${ }_{0} b_{p}^{*}$ is the optimal pure periodic barrier. The detailed method of deriving $G_{P}\left(x ;{ }_{0} b_{p}^{*}\right)$ without proportional costs can be found in Albrecher et al. (2011b). By adapting the results from Albrecher et al. (2011b), we have

$$
\begin{aligned}
& G_{P}\left(x ;{ }_{0} b_{p}^{*}\right) \\
& = \begin{cases}\eta_{p}\left(\frac{e^{r_{0} x}-e^{s_{0} x}}{r_{0} e^{r_{0}\left(b_{p}^{*}\right)}-s_{0} e^{s_{0}\left(b_{p}^{*}\right)}}\right), & x \in\left[0,{ }_{0} b_{p}^{*}\right), \\
\eta_{p}\left[\frac{\delta}{\gamma+\delta} \frac{e^{s_{\gamma}\left(x-0 b_{p}^{*}\right)}}{s_{\gamma}}+\frac{\gamma}{\gamma+\delta}\left(\frac{\mu}{\gamma+\delta}+\left(x-{ }_{0} b_{p}^{*}\right)\right)\right] & \\
+\frac{\gamma}{\gamma+\delta} G_{P}\left({ }_{0} b_{p}^{*} ;{ }_{0} b_{p}^{*}\right), & x \in\left[{ }_{0} b_{p}^{*}, \infty\right),\end{cases}
\end{aligned}
$$




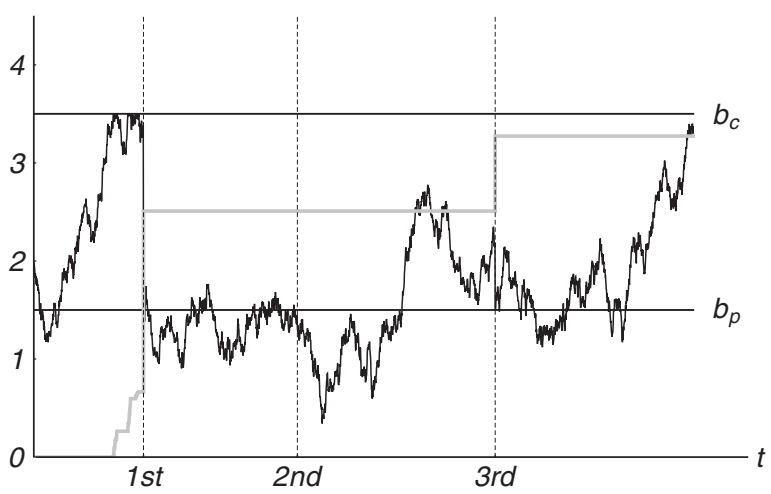

FIGURE 1: Sample paths of $X(t)$ and $D(t)$ under a hybrid barrier dividend strategy.

where the optimal periodic barrier has the following explicit form:

$$
{ }_{0} b_{p}^{*}=\frac{1}{r_{0}-s_{0}} \log \left(\frac{s_{0}\left[(\gamma+\delta) s_{0}-s_{\gamma} \delta\right]}{r_{0}\left[(\gamma+\delta) r_{0}-s_{\gamma} \delta\right]}\right),
$$

and the root $s_{\gamma}$ is defined in (3.12). The function $G_{P}\left(x ;{ }_{0} b_{p}^{*}\right)$ is strictly increasing and concave with the following properties:

$$
G_{P}^{\prime}\left({ }_{0} b_{p}^{*} ;{ }_{0} b_{p}^{*}\right)=\eta_{p} \quad \text { and } \quad \lim _{x \rightarrow \infty} G_{P}^{\prime}\left(x ;{ }_{0} b_{p}^{*}\right)=\eta_{p} \frac{\gamma}{\gamma+\delta} ;
$$

see Avanzi et al. (2014).

\section{EXPECTED PRESENT VALUE OF DIVIDENDS UNDER AN OPTIMAL HYBRID BARRIER STRATEGY}

In this section, we focus on a specific class of hybrid strategies - the hybrid barrier strategies. In a hybrid barrier strategy, dividends can be distributed according to a periodic and a continuous barrier strategy. The periodic barrier is assumed to be strictly less than the continuous barrier. In Figure 1, we plot a simulated sample path of the modified surplus process $\{X(t), t \geq 0\}$ and the corresponding dividend process $\{D(t), t \geq 0\}$. There, three decision times occur. Prior to the first dividend decision time, the surplus reaches the continuous barrier level $\left(b_{c}\right)$ and all the excess surplus is continuously distributed as dividends. At the first and third (periodic) dividend decision times, lump sum dividend payments are made to bring the surplus down to the periodic barrier level $\left(b_{p}\right)$. At the second periodic dividend decision time, the surplus is below the periodic barrier level and hence no dividend is paid.

In the next two sections, we first consider an optimal hybrid barrier strategy without liquidation in Section 3.1, where both barriers are strictly positive and finite $\left(0<b_{p}^{*}<b_{c}^{*}<\infty\right)$. In Section 3.2, we allow the optimal periodic barrier 
to be zero $\left(0=b_{p}^{*}<b_{c}^{*}<\infty\right)$ and this is referred as an optimal hybrid barrier strategy with liquidation-at-first-periodic-opportunity. Throughout Section 3, we assume that proportional transaction costs on periodic dividends are strictly less than proportional costs on continuous dividends $\left(\eta_{c}<\eta_{p}\right)$. In Section 4, we show that this assumption forms the necessary and sufficient condition for optimal hybrid strategies to be optimal in the set of hybrid strategies.

\subsection{When liquidation is never optimal}

Suppose we follow an optimal hybrid barrier strategy with strictly positive and finite barriers, that is, when $0<b_{p}^{*}<b_{c}^{*}<\infty$. The expected present value of dividends until ruin $G_{H}\left(x ; b_{p}^{*}, b_{c}^{*}\right)$ is constituted of three layers,

$$
G_{H}\left(x ; b_{p}^{*}, b_{c}^{*}\right)= \begin{cases}G_{L}(x), & x \in\left[0, b_{p}^{*}\right), \\ G_{M}(x), & x \in\left[b_{p}^{*}, b_{c}^{*}\right), \\ G_{U}(x), & x \in\left[b_{c}^{*}, \infty\right) .\end{cases}
$$

We start by determining differential equations that $G_{H}\left(x ; b_{p}^{*}, b_{c}^{*}\right)$ must satisfy. Consider a small time interval $[0, h)$ and if the surplus level $x \in\left[0, b_{p}^{*}\right)$, then no dividends can be issued and we have

$$
G_{L}(x)=(1-\delta h) \mathbb{E}\left[G_{L}(x+\mu h+\sigma W(h))\right]+o(h) .
$$

Thanks to the properties of the Brownian motion, we can expand the expectation in (3.2) to

$$
\mathbb{E}\left[G_{L}(x+\mu h+\sigma W(h))\right]=G_{L}(x)+\mu G_{L}^{\prime}(x) h+\frac{\sigma^{2}}{2} G_{L}^{\prime \prime}(x) h+o(h) .
$$

Substituting (3.3) into (3.2), dividing $h$ on both sides and letting $h \rightarrow 0$ yields the following differential equation for $G_{L}(x)$ :

$$
\frac{\sigma^{2}}{2} G_{L}^{\prime \prime}(x)+\mu G_{L}^{\prime}(x)-\delta G_{L}(x)=0, \quad \text { for } \quad x \in\left[0, b_{p}^{*}\right) .
$$

If the surplus level is zero, then ruin is immediate and no dividends are paid. Therefore we have $G_{L}(0)=0$.

When the surplus level is above $b_{p}^{*}$ and strictly below $b_{c}^{*}$, then no continuous dividends are issued and periodic dividends are issued according to a periodic barrier strategy. Then $G_{M}(x)$ can be expressed by conditioning on the arrival of periodic dividends during the time interval $[0, h)$, i.e.,

$$
\begin{aligned}
G_{M}(x)= & \gamma h(1-\delta h)\left\{\eta_{p}\left(x-b_{p}^{*}\right)+\mathbb{E}\left[G_{M}\left(b_{p}^{*}+\mu h+\sigma W(h)\right)\right]\right\} \\
& +(1-\gamma h)(1-\delta h) \times \mathbb{E}\left[G_{M}(x+\mu h+\sigma W(h))\right]+o(h) .
\end{aligned}
$$


If we expand the expectations using (3.3), divide $h$ to both sides of (3.5) and take $h \rightarrow 0$, we obtain

$$
\begin{aligned}
& \frac{\sigma^{2}}{2} G_{M}^{\prime \prime}(x)+\mu G_{M}^{\prime}(x)-\delta G_{M}(x)+\gamma\left[\eta_{p}\left(x-b_{p}^{*}\right)+G_{L}\left(b_{p}^{*}\right)-G_{M}(x)\right] \\
& \quad=0, \quad x \in\left[b_{p}^{*}, b_{c}^{*}\right) .
\end{aligned}
$$

Finally, if the surplus is above $b_{c}^{*}$, all excess surplus above $b_{c}^{*}$ is paid out immediately as continuous dividends and $G_{U}(x)$ satisfies a linear equation

$$
G_{U}(x)=\eta_{c}\left(x-b_{c}^{*}\right)+G_{M}\left(b_{c}^{*}\right), \quad x \in\left[b_{c}^{*}, \infty\right) .
$$

It can be shown that $G_{H}\left(x ; b_{p}^{*}, b_{c}^{*}\right)$ and $G_{H}^{\prime}\left(x ; b_{p}^{*}, b_{c}^{*}\right)$ are continuous functions of $x$. First, $G_{H}\left(x ; b_{p}^{*}, b_{c}^{*}\right)$ is continuous at $x=b_{c}^{*}$ by construction of a continuous barrier strategy. We can substitute $x=b_{c}^{*}$ into (3.7) and have $G_{U}\left(b_{c}^{*}\right)=G_{M}\left(b_{c}^{*}\right)$. To show that $G_{H}^{\prime}\left(x ; b_{p}^{*}, b_{c}^{*}\right)$ is continuous at $b_{c}^{*}$, we consider the following two scenarios, $x=b_{c}^{*}$ and $x=b_{c}^{*}-\epsilon$. In the second scenario, the surplus reaches the barrier $b_{c}^{*}$ after an instant due to oscillation from the Brownian motion. In such a small instant, the chance to have a periodic dividend decision is too small for any periodic dividends to be distributed. In the first scenario, a dividend of $\epsilon$ is distributed. Therefore, the expected present value of dividends in the first scenario is higher than the second scenario by an amount of $\eta_{c} \epsilon$ for small $\epsilon$. It follows that

$$
G_{U}\left(b_{c}^{*}\right)=G_{M}\left(b_{c}^{*}\right)=G_{M}\left(b_{c}^{*}-\epsilon\right)+\eta_{c} \epsilon \Longrightarrow G_{M}^{\prime}\left(b_{c}^{*}\right)=\eta_{c},
$$

by dividing $\epsilon$ and taking $\epsilon \rightarrow 0$. By adopting a similar argument discussed in Zhang and Cheung (2014), we can show that $G_{H}\left(x ; b_{p}^{*} ; b_{c}^{*}\right)$ and $G_{H}^{\prime}\left(x ; b_{p}^{*} ; b_{c}^{*}\right)$ are continuous with some help from fluctuation theory. Now, when the optimal periodic and continuous barriers $b_{p}^{*}$ and $b_{c}^{*}$ are applied, we have two additional conditions (only for the optimal barriers)

$$
G_{H}^{\prime}\left(b_{p}^{*}-; b_{p}^{*} ; b_{c}^{*}\right)=G_{H}^{\prime}\left(b_{p}^{*}+; b_{p}^{*} ; b_{c}^{*}\right)=\eta_{p} \quad \text { and } \quad G_{H}^{\prime \prime}\left(b_{c}^{*}-; b_{p}^{*} ; b_{c}^{*}\right)=0 .
$$

The justification of the first condition can be found in Lemma 3.1 from Avanzi et al. (2014). The second condition $G_{H}^{\prime \prime}\left(x ; b_{p}^{*} ; b_{c}^{*}\right)=0$ is due to smooth pasting.

Remark 3.1. Suppose that $G_{H}\left(x ; b_{p}^{*}, b_{c}^{*}\right)$ is an increasing and concave function, then we have

$$
G_{H}^{\prime}\left(b_{p}^{*} ; b_{p}^{*}, b_{c}^{*}\right)=\eta_{p}>\eta_{c}=G_{H}^{\prime}\left(b_{c}^{*} ; b_{p}^{*}, b_{c}^{*}\right) \Longleftrightarrow b_{p}^{*}<b_{c}^{*} .
$$

This suggests that $\eta_{p}>\eta_{c}$ is necessary and sufficient for an optimal hybrid barrier strategy to exist. This also means that $\eta_{c} \geq \eta_{p} \Longleftrightarrow b_{c}^{*} \leq b_{p}^{*}$. Note that a hybrid barrier strategies with $b_{c} \leq b_{p}$ is equivalent to a pure continuous barrier strategy since the surplus is always upper-bounded by the continuous barrier $b_{c}$. This suggests that if the proportional transaction costs on continuous dividends satisfy $\eta_{c} \in\left[\eta_{p}, 1\right]$ (continuous dividends are cheaper than periodic 
dividends), the pure continuous barrier strategy is (unsurprisingly) the optimal hybrid strategy; see Section 4.5 for a proof.

Now we solve the differential equations (3.4) and (3.6) to obtain explicit solution of $G_{H}\left(x ; b_{p}^{*}, b_{c}^{*}\right)$. We first recognise that (3.4) is a linear second-order ordinary differential equation and has general solution

$$
G_{L}(x)=K_{1} e^{r_{0} x}+K_{2} e^{s_{0} x}, \quad \text { for } \quad x \in\left[0, b_{p}^{*}\right),
$$

where $K_{1}$ and $K_{2}$ are some constants, and where $r_{\gamma}>0$ and $s_{\gamma}<0$ are the roots (as functions of $\gamma$ ) of the characteristic equation

$$
\frac{\sigma^{2}}{2} \xi^{2}+\mu \xi-(\delta+\gamma)=0
$$

Next, we solve (3.6) by recognising that it is a non-homogeneous linear second order differential equation. The general solution consists of a homogeneous and a particular solution. The homogeneous solution is $C_{1} e^{r_{\gamma} x}+C_{2} e^{s_{\gamma} x}$. A particular solution to (3.6) is in the form of $A x+B$. If we substitute this particular solution back into (3.6), we obtain

$$
A=\frac{\eta_{p} \gamma}{\gamma+\delta} \quad \text { and } \quad B=\frac{\eta_{p} \mu \gamma}{(\gamma+\delta)^{2}}+\frac{\left(G_{L}\left(b_{p}^{*}\right)-\eta_{p} b_{p}^{*}\right) \gamma}{\gamma+\delta} .
$$

Hence, a general solution of (3.6) is

$$
\begin{gathered}
G_{M}(x)=C_{1} e^{r_{\gamma} x}+C_{2} e^{s_{\gamma} x}+\frac{\gamma}{\gamma+\delta}\left[\frac{\eta_{p} \mu}{\gamma+\delta}+\eta_{p}\left(x-b_{p}^{*}\right)+G_{L}\left(b_{p}^{*}\right)\right], \\
\text { for } x \in\left[b_{p}^{*}, b_{c}^{*}\right) .
\end{gathered}
$$

To completely specify $G_{H}\left(x ; b_{p}^{*}, b_{c}^{*}\right)$, we need to find the constants $K_{1}$ and $K_{2}$ in (3.11), the constants $C_{1}$ and $C_{2}$ in (3.14), as well as the two barriers $b_{p}^{*}$ and $b_{c}^{*}$. We use the initial condition $G_{H}\left(0 ; b_{p}^{*}, b_{c}^{*}\right)=0$ and the fact that $G_{H}\left(x ; b_{p}^{*}, b_{c}^{*}\right)$ and $G_{H}^{\prime}\left(x ; b_{p}^{*}, b_{c}^{*}\right)$ are continuous functions to obtain the following equations:

$$
\begin{aligned}
G_{L}(0)=0 \Longrightarrow K_{1}+K_{2}=0 ; \\
G_{L}\left(b_{p}^{*}\right)=G_{M}\left(b_{p}^{*}\right) \Longrightarrow K_{1} e^{r_{0} b_{p}^{*}}+K_{2} e^{s_{0} b_{p}^{*}} \\
=\frac{\gamma+\delta}{\delta}\left(C_{1} e^{r_{\gamma} b_{p}^{*}}+C_{2} e^{s_{\gamma} b_{p}^{*}}\right)+\frac{\gamma}{\gamma+\delta} \frac{\eta_{p} \mu}{\delta} \\
G_{L}^{\prime}\left(b_{p}^{*}\right)=G_{M}^{\prime}\left(b_{p}^{*}\right) \Longrightarrow K_{1} r_{0} e^{r_{0} b_{p}^{*}}+K_{2} s_{0} e^{s_{0} b_{p}^{*}} \\
=C_{1} r_{\gamma} e^{r_{\gamma} b_{p}^{*}}+C_{2} s_{\gamma} e^{s_{\gamma} b_{p}^{*}}+\frac{\eta_{p} \gamma}{\gamma+\delta} ; \\
G_{M}^{\prime}\left(b_{c}^{*}\right)=G_{U}^{\prime}\left(b_{c}^{*}\right) \Longrightarrow C_{1} r_{\gamma} e^{r_{\gamma} b_{c}^{*}}+C_{2} s_{\gamma} e^{s_{\gamma} b_{c}^{*}}+\frac{\eta_{p} \gamma}{\gamma+\delta}=\eta_{c} .
\end{aligned}
$$


According to (3.9), the two following additional conditions are satisfied when the optimal barriers $b_{p}^{*}$ and $b_{c}^{*}$ are applied:

$$
\begin{aligned}
G_{H}^{\prime}\left(b_{p}^{*} ; b_{p}^{*}, b_{c}^{*}\right)=\eta_{p} & \Longrightarrow G_{M}^{\prime}\left(b_{p}^{*}\right)= \\
\eta_{p} & \Longrightarrow C_{1} r_{\gamma} e^{r_{\gamma} b_{p}^{*}}+C_{2} s_{\gamma} e^{s_{\gamma} b_{p}^{*}}+\frac{\eta_{p} \gamma}{\gamma+\delta}=\eta_{p} ; \\
G_{M}^{\prime \prime}\left(b_{c}^{*}\right)=G_{U}^{\prime \prime}\left(b_{c}^{*}\right) & \Longrightarrow G_{M}^{\prime \prime}\left(b_{c}^{*}\right)= \\
0 & \Longrightarrow C_{1} r_{\gamma}^{2} e^{r_{\gamma} b_{c}^{*}}+C_{2} s_{\gamma}^{2} e^{s_{\gamma} b_{c}^{*}}=0 .
\end{aligned}
$$

Note that the optimality condition in (3.19), combined with the fact that $G_{H}^{\prime}\left(x ; b_{p}^{*}, b_{c}^{*}\right)$ is continuous everywhere, leads to two equivalent equations, $G_{L}^{\prime}\left(b_{p}^{*}\right)=\eta_{p}$ or $G_{M}^{\prime}\left(b_{p}^{*}\right)=\eta_{p}$. We choose $G_{M}^{\prime}\left(b_{p}^{*}\right)=\eta_{p}$ for the ease of solving for $G_{H}\left(x ; b_{p}^{*}, b_{c}^{*}\right)$.

Remark 3.2. Solving the six equations (3.15)-(3.20) completely determines all six unknown parameters $K_{1}, K_{2}, C_{1}, C_{2}, b_{p}^{*}$ and $b_{c}^{*}$. Equations (3.15)-(3.18) are sufficient to determine the four constants $K_{1}, K_{2}, C_{1}$ and $C_{2}$ for arbitrary barriers $b_{p}$ and $b_{c}$.

We now derive an explicit solution of $G_{H}\left(x ; b_{p}^{*}, b_{c}^{*}\right)$. First we can solve (3.18) and (3.20) simultaneously for coefficients $C_{1}$ and $C_{2}$,

$$
\begin{aligned}
C_{1} & =\left(\eta_{c}-\eta_{p} \frac{\gamma}{\gamma+\delta}\right) \frac{e^{-r_{\gamma} b_{c}^{*}}}{r_{\gamma}} \frac{s_{\gamma}}{s_{\gamma}-r_{\gamma}} \text { and } \\
C_{2} & =\left(\eta_{c}-\eta_{p} \frac{\gamma}{\gamma+\delta}\right) \frac{e^{-s_{\gamma} b_{c}^{*}}}{s_{\gamma}} \frac{r_{\gamma}}{r_{\gamma}-s_{\gamma}} .
\end{aligned}
$$

Substituting $C_{1}$ and $C_{2}$ back into (3.14) gives

$$
\begin{aligned}
G_{M}(x)=( & \left.\eta_{c}-\eta_{p} \frac{\gamma}{\gamma+\delta}\right)\left[\frac{s_{\gamma}}{s_{\gamma}-r_{\gamma}} \frac{e^{r_{\gamma}\left(x-b_{c}^{*}\right)}}{r_{\gamma}}+\frac{r_{\gamma}}{r_{\gamma}-s_{\gamma}} \frac{e^{s_{\gamma}\left(x-b_{c}^{*}\right)}}{s_{\gamma}}\right] \\
& +\frac{\gamma}{\gamma+\delta}\left[\frac{\eta_{p} \mu}{\gamma+\delta}+\eta_{p}\left(x-b_{p}^{*}\right)+G_{L}\left(b_{p}^{*}\right)\right] .
\end{aligned}
$$

This is a semi-explicit solution for $G_{M}(x)$ as $b_{p}^{*}$ and $b_{c}^{*}$ are still unknown at this point. Next we substitute $C_{1}$ and $C_{2}$ in (3.21) into (3.19) to obtain the following equation:

$$
\left(\eta_{c}-\eta_{p} \frac{\gamma}{\gamma+\delta}\right)\left[\frac{s_{\gamma}}{s_{\gamma}-r_{\gamma}} e^{-r_{\gamma}\left(b_{c}^{*}-b_{p}^{*}\right)}+\frac{r_{\gamma}}{r_{\gamma}-s_{\gamma}} e^{-s_{\gamma}\left(b_{c}^{*}-b_{p}^{*}\right)}\right]=\eta_{p} \frac{\delta}{\gamma+\delta} .
$$


The trick here is to solve for $b_{c}^{*}-b_{p}^{*}$ in (3.23), which is equivalent to finding the root of

$$
h(\xi)=\left(\eta_{c}-\eta_{p} \frac{\gamma}{\gamma+\delta}\right)\left(\frac{s_{\gamma}}{s_{\gamma}-r_{\gamma}} e^{-r_{\gamma} \xi}+\frac{r_{\gamma}}{r_{\gamma}-s_{\gamma}} e^{-s_{\gamma} \xi}\right)-\eta_{p} \frac{\delta}{\gamma+\delta} .
$$

Because $b_{c}^{*}-b_{p}^{*}>0$ here, the natural domain for $h(\xi)$ is $\xi \in(0, \infty)$. By considering the sign of $h^{\prime}(\xi)$, a sufficient and necessary condition for $h(\xi)$ to have a unique positive root is (see details in Section A.1)

$$
\begin{aligned}
& \eta_{c}-\eta_{p} \frac{\gamma}{\gamma+\delta} \leq 0 \Longleftrightarrow h(\xi) \text { has no root; } \\
& \eta_{c}-\eta_{p} \frac{\gamma}{\gamma+\delta}>0 \Longleftrightarrow h(\xi) \text { has a unique positive root. }
\end{aligned}
$$

Condition (3.26) is a necessary and sufficient condition for $b_{c}^{*}-b_{p}^{*}$ to exist. Combining (3.26) with Remark 3.1 reveals that the necessary and sufficient condition for an optimal hybrid barrier strategies to exist is $\eta_{c} \in\left(\eta_{p} \frac{\gamma}{\gamma+\delta}, \eta_{p}\right)$. We prove this statement using a verification theorem in Section 4.3.

Remark 3.3. Because of (3.26), a pure periodic dividend strategy will be optimal if and only if

$$
\eta_{c} \leq \eta_{p} \frac{\gamma}{\gamma+\delta}
$$

Note that the right-hand side of (3.27) corresponds to the expected present value of a payment of $\eta_{p}$ at the next dividend decision time. It makes sense that this quantity should be higher than $\eta_{c}$ (the value of an immediate payment). If not, it would never make sense to wait and pay under the umbrella of the periodic strategy. This is formally shown in Section 4.4.

Next, using the explicit form of $G_{M}(x)$ in (3.22), we can substitute $x=b_{p}^{*}$ and obtain

$$
\begin{aligned}
G_{M}\left(b_{p}^{*}\right)= & \frac{\eta_{p} \mu}{\delta} \frac{\gamma}{\gamma+\delta}+\left(\frac{\gamma+\delta}{\delta}\right)\left(\eta_{c}-\eta_{p} \frac{\gamma}{\gamma+\delta}\right) \\
& \times\left[\frac{s_{\gamma}}{s_{\gamma}-r_{\gamma}} \frac{e^{-r_{\gamma}\left(b_{c}^{*}-b_{p}^{*}\right)}}{r_{\gamma}}+\frac{r_{\gamma}}{r_{\gamma}-s_{\gamma}} \frac{e^{-s_{\gamma}\left(b_{c}^{*}-b_{p}^{*}\right)}}{s_{\gamma}}\right],
\end{aligned}
$$

where $b_{c}^{*}-b_{p}^{*}$ is the solution to (3.23). This quantity is needed to solve $G_{L}(x)$.

Remark 3.4. We can simplify (3.28) by using (3.23) and obtain an alternative form of $G_{M}\left(b_{p}^{*}\right)$,

$$
G_{M}\left(b_{p}^{*}\right)=\eta_{p}\left(\frac{\mu}{\delta} \frac{\gamma}{\gamma+\delta}+\frac{1}{s_{\gamma}}\right)+\left(\frac{\gamma+\delta}{\delta}\right)\left(\eta_{c}-\eta_{p} \frac{\gamma}{\gamma+\delta}\right) \frac{e^{-r_{\gamma}\left(b_{c}^{*}-b_{p}^{*}\right)}}{r_{\gamma}} .
$$


The expression in the first brackets of (3.29) is the expected present value of dividends in a pure periodic barrier strategy $G_{P}\left({ }_{0} b_{p}^{*} ;{ }_{0} b_{p}^{*}\right)$ without proportional transaction costs (see, e.g., (31) in Albrecher et al., 2011b). The remaining terms in (3.29) can be interpreted as additional dividends received from hybrid continuous dividends, which goes to zero when the pure periodic dividend strategies become optimal; that is, when $b_{c}^{*} \rightarrow \infty$; see also illustrations in Section 5.

We now proceed to obtain an explicit solution for $G_{L}(x)$. Using (3.15) and combining (3.17) and (3.19), we have

$$
\begin{aligned}
G_{L}^{\prime}\left(b_{p}^{*}\right) & =\eta_{p} \Longrightarrow K_{1} r_{0} e^{r_{0} b_{p}^{*}}+K_{2} s_{0} e^{s_{0} b_{p}^{*}}=\eta_{p} ; \\
G_{L}(0) & =0 \Longrightarrow K_{1}+K_{2}=0 .
\end{aligned}
$$

Solving the equations simultaneously yields $K_{1}$ and $K_{2}$. Substituting $K_{1}$ and $K_{2}$ into $G_{L}(x)$ gives

$$
G_{L}(x)=\eta_{p} \frac{e^{r_{0} x}-e^{s_{0} x}}{r_{0} e^{r_{0} b_{p}^{*}}-s_{0} e^{s_{0} b_{p}^{*}}}, \quad x \in\left[0, b_{p}^{*}\right) .
$$

Here, the function $G_{L}(x)$ is not completely specified as $b_{p}^{*}$ is still unknown. Using $G_{M}\left(b_{p}^{*}\right)$ in (3.28) (which is independent of $\left.b_{p}^{*}\right)$ and $G_{L}\left(b_{p}^{*}\right)=G_{M}\left(b_{p}^{*}\right)$, we have

$$
\begin{aligned}
& G_{L}\left(b_{p}^{*}\right)=\eta_{p} \frac{e^{r_{0} b_{p}^{*}}-e^{s_{0} b_{p}^{*}}}{r_{0} e^{r_{0} b_{p}^{*}}-s_{0} e^{s_{0} b_{p}^{*}}}=G_{M}\left(b_{p}^{*}\right) \\
& \Longleftrightarrow b_{p}^{*}=\frac{1}{r_{0}-s_{0}} \log \left(\frac{\eta_{p}-s_{0} G_{M}\left(b_{p}^{*}\right)}{\eta_{p}-r_{0} G_{M}\left(b_{p}^{*}\right)}\right) .
\end{aligned}
$$

Remark 3.5. The lower branch of $G_{H}\left(x ; b_{p}^{*}, b_{c}^{*}\right)=G_{L}(x)$ in (3.32) has a similar form to both $G_{C}\left(x ;{ }_{0} b_{c}^{*}\right)$ in (2.9) and $G_{P}\left(x ;{ }_{0} b_{p}^{*}\right)$ in (2.11) below their respective barrier levels. This similarity arises from the fact that when the surplus is below the hybrid periodic barrier $x \in\left[0, b_{p}^{*}\right)$, no dividends are issued until the surplus reaches $b_{p}^{*}$, regardless of the arrival of periodic dividend decisions. One can use the Markov property of the surplus process at the first hitting time of reaching $b_{p}^{*}$ before ruin time $\tau$ to obtain $G_{L}(x)$ (see Kyprianou, 2006, Section 8.2). The expected present value of a dollar paid at the first time the process hits some higher level has the same functional form here than in $G_{C}\left(x ;{ }_{0} b_{c}^{*}\right)$ for $x \in\left[0,{ }_{0} b_{c}^{*}\right)$ and $G_{P}\left(x ;{ }_{0} b_{p}^{*}\right)$ for $x \in\left[0,{ }_{0} b_{p}^{*}\right)$, which explains their similarity.

The optimal periodic barrier $b_{p}^{*}$ is available in closed form in (3.33), but this solution may not always be strictly positive. Note that in the first part of (3.33) $r_{0} e^{r_{0} b_{p}^{*}}-s_{0} e^{s_{0} b_{p}^{*}}$ and $e^{r_{0} b_{p}^{*}}-e^{s_{0} b_{p}^{*}}$ are both strictly positive for $b_{p}^{*}>0$. So a strictly positive solution $b_{p}^{*}$ exists if and only if $G_{M}\left(b_{p}^{*}\right)>0-$ note that $G_{M}\left(b_{p}^{*}\right)$ in (3.28) does not require $b_{p}^{*}$. In fact, when

$$
G_{H}\left(b_{p}^{*} ; b_{p}^{*}, b_{c}^{*}\right) \leq 0,
$$


then the optimal hybrid strategy becomes a hybrid barrier strategy with liquidation-at-first-periodic-opportunity $\left(0=b_{p}^{*}<b_{c}^{*}<\infty\right)$; this is shown in Section 4.3.1. We will refer to (3.34) as the insufficient-prospect condition. In the next section, we consider the liquidation-at-first-periodic-opportunity strategy in detail and derive its expected present value of dividends $G_{H}\left(x ; 0, b_{c}^{*}\right)$. We discuss the different situations that lead to the insufficient-prospect condition in Section 5.4.

Remark 3.6. In a pure optimal periodic barrier strategy, the insufficientprospect condition is also characterised by the expected present value of dividends until ruin when the surplus is evaluated at the optimal periodic barrier level (see, for example, equation (4.46) in Avanzi et al., 2014).

\subsection{When a liquidation-at-first-periodic-opportunity is optimal}

Consider now the case where the periodic barrier is zero, but where a strictly positive continuous barrier exists $\left(0=b_{p}^{*}<b_{c}^{*}<\infty\right)$. Under such a strategy, dividends are distributed according to a continuous barrier strategy, but the company is liquidated at the first periodic dividend payment opportunity. We call this a hybrid barrier strategy with liquidation-at-first-periodic-opportunity. This happens when business is not really profitable (the insufficient-prospect condition), but transaction costs are so high within the continuous framework that it is better to wait and liquidate when this is done at lower cost (at the first periodic opportunity). In this case, the expected present value of dividends until ruin $G_{H}\left(x ; 0, b_{c}^{*}\right)$ can be separated into two layers:

$$
G_{H}\left(x ; 0, b_{c}^{*}\right)=\left\{\begin{array}{l}
F_{L}(x), x \in\left[0, b_{c}^{*}\right), \\
F_{U}(x), x \in\left[b_{c}^{*}, \infty\right) .
\end{array}\right.
$$

We can substitute $b_{p}^{*}=0$ in (3.6) and obtain

$$
\frac{\sigma^{2}}{2} F_{L}^{\prime \prime}(x)+\mu F_{L}^{\prime}(x)-\delta F_{L}(x)+\gamma\left[\eta_{p} x-F_{L}(x)\right]=0, \quad x \in\left[0, b_{c}^{*}\right) .
$$

Similar to $G_{U}(x)$ in (3.7), $F_{U}(x)$ satisfies

$$
F_{U}(x)=\eta_{c}\left(x-b_{c}^{*}\right)+F_{L}\left(b_{c}^{*}\right), \quad \text { for } \quad x \in\left[b_{c}^{*}, \infty\right) .
$$

Since ruin occurs at $x=0$, a boundary condition is $F_{L}(0)=0$. Similar to $G_{H}\left(x ; b_{p}^{*}, b_{c}^{*}\right)$, we also require a first-order continuity condition $F_{L}^{\prime}\left(b_{c}^{*}\right)=$ $\eta_{c}$. The optimality condition for $b_{c}^{*}$ is the second-order continuity condition $F_{L}^{\prime \prime}\left(b_{c}^{*}\right)=0$.

A general solution for $F_{L}(x)$ stems from the second-order inhomogeneous equation in (3.36),

$$
F_{L}(x)=A e^{r_{\gamma} x}+B e^{s_{\gamma} x}+\frac{\eta_{p} \gamma}{\gamma+\delta}\left(x+\frac{\mu}{\gamma+\delta}\right), \quad \text { for } x \in\left[0, b_{c}^{*}\right) .
$$


Using the conditions $F_{L}^{\prime}\left(b_{c}^{*}\right)=\eta_{c}$ and $F_{L}^{\prime \prime}\left(b_{c}^{*}\right)=0$, we have

$$
\begin{aligned}
& F_{L}^{\prime}\left(b_{c}^{*}\right)=\eta_{c} \Longrightarrow A r_{\gamma} e^{r_{\gamma} b_{c}^{*}}+B s_{\gamma} e^{s_{\gamma} b_{c}^{*}}=\eta_{c}-\eta_{p} \frac{\gamma}{\gamma+\delta} \text { and } \\
& F_{L}^{\prime \prime}\left(b_{c}^{*}\right)=0 \Longrightarrow A r_{\gamma}^{2} e^{r_{\gamma} b_{c}^{*}}+B s_{\gamma}^{2} e^{s_{\gamma} b_{c}^{*}}=0 .
\end{aligned}
$$

Solving $A$ and $B$ simultaneously and substituting them back into $F_{L}(x)$ yields

$$
\begin{gathered}
F_{L}(x)=\left(\eta_{c}-\eta_{p} \frac{\gamma}{\gamma+\delta}\right)\left(\frac{s_{\gamma}}{s_{\gamma}-r_{\gamma}} \frac{e^{r_{\gamma}\left(x-b_{c}^{*}\right)}}{r_{\gamma}}+\frac{r_{\gamma}}{r_{\gamma}-s_{\gamma}} \frac{e^{s_{\gamma}\left(x-b_{c}^{*}\right)}}{s_{\gamma}}\right) \\
+\frac{\eta_{p} \gamma}{\gamma+\delta}\left(x+\frac{\mu}{\gamma+\delta}\right), \quad \text { for } x \in\left[0, b_{c}^{*}\right),
\end{gathered}
$$

which is very similar to $G_{M}(x)$ in (3.22). Next, we use the condition $F_{L}(0)=0$ to find $b_{c}^{*}$. The continuous barrier $b_{c}^{*}$ is then the solution of

$$
\left(\eta_{c}-\eta_{p} \frac{\gamma}{\gamma+\delta}\right)\left(\frac{s_{\gamma}}{s_{\gamma}-r_{\gamma}} \frac{e^{-r_{\gamma} b_{c}^{*}}}{r_{\gamma}}+\frac{r_{\gamma}}{r_{\gamma}-s_{\gamma}} \frac{e^{-s_{\gamma} b_{c}^{*}}}{s_{\gamma}}\right)+\frac{\eta_{p} \gamma}{\gamma+\delta} \frac{\mu}{\gamma+\delta}=0 .
$$

In Section A.3, we show that a unique and positive $b_{c}^{*}$ exists if and only if the proportional costs satisfy $\eta_{c}>\eta_{p} \gamma / \gamma+\delta$ and the drift $(\mu)$ is strictly positive. In fact, the two conditions with the insufficient-prospect condition are key to show the optimality of the optimal hybrid barrier strategy with liquidation-at-first-periodic-opportunity; the formal proof is provided in Section 4.3.1.

Remark 3.7. Note that in an optimal hybrid barrier strategy without liquidation, we find $b_{c}^{*}-b_{p}^{*}$ via (3.23) and $b_{p}^{*}$ via (3.33) to specify both barriers. In a liquidation-at-first-periodic-opportunity strategy, only $b_{c}^{*}$ is required and is obtained by solving (3.42).

\subsection{Summary}

If the proportional transaction costs on dividends issued continuously are at a level such that $\eta_{c} \in\left(\eta_{p} \frac{\gamma}{\gamma+\delta}, \eta_{p}\right)$ and the insufficient-prospect condition (3.34) does not hold, the expected present value of dividends for an optimal hybrid 
barrier strategy $G_{H}\left(x ; b_{p}^{*}, b_{c}^{*}\right)$ is

$$
\begin{aligned}
G_{L}(x)= & \eta_{p} \frac{e^{r_{0} x}-e^{s_{0} x}}{r_{0} e^{r_{0} b_{p}^{*}}-s_{0} e^{s_{0} b_{p}^{*}}}, \quad \text { for } x \in\left[0, b_{p}^{*}\right) ; \\
G_{M}(x)= & \left(\eta_{c}-\eta_{p} \frac{\gamma}{\gamma+\delta}\right)\left[\frac{s_{\gamma}}{s_{\gamma}-r_{\gamma}} \frac{e^{r_{\gamma}\left(x-b_{c}^{*}\right)}}{r_{\gamma}}+\frac{r_{\gamma}}{r_{\gamma}-s_{\gamma}} \frac{e^{s_{\gamma}\left(x-b_{c}^{*}\right)}}{s_{\gamma}}\right] \\
& +\frac{\gamma}{\gamma+\delta}\left[\frac{\eta_{p} \mu}{\gamma+\delta}+\eta_{p}\left(x-b_{p}^{*}\right)+G_{L}\left(b_{p}^{*}\right)\right], \\
& \text { for } x \in\left[b_{p}^{*}, b_{c}^{*}\right) ; \text { and } \\
G_{U}(x)= & \eta_{c}\left(x-b_{c}^{*}\right)+G_{M}\left(b_{c}^{*}\right), \quad \text { for } x \in\left[b_{c}^{*}, \infty\right),
\end{aligned}
$$

where $b_{c}^{*}-b_{p}^{*}$ is the unique positive root to the equation

$$
\left(\eta_{c}-\eta_{p} \frac{\gamma}{\gamma+\delta}\right)\left[\frac{s_{\gamma}}{s_{\gamma}-r_{\gamma}} e^{-r_{\gamma}\left(b_{c}^{*}-b_{p}^{*}\right)}+\frac{r_{\gamma}}{r_{\gamma}-s_{\gamma}} e^{-s_{\gamma}\left(b_{c}^{*}-b_{p}^{*}\right)}\right]=\eta_{p} \frac{\delta}{\gamma+\delta},
$$

and where $b_{p}^{*}$ is

$$
b_{p}^{*}=\frac{1}{r_{0}-s_{0}} \log \left(\frac{\eta_{p}-s_{0} G_{M}\left(b_{p}^{*}\right)}{\eta_{p}-r_{0} G_{M}\left(b_{p}^{*}\right)}\right) .
$$

If the proportional transaction costs on dividends issued continuously are at a level such that $\eta_{c} \in\left(\eta_{p} \frac{\gamma}{\gamma+\delta}, \eta_{p}\right)$ and the insufficient-prospect condition (3.34) does hold, then the optimal periodic barrier is zero. The expected present value of dividends of an optimal hybrid barrier strategy with liquidation-at-firstperiodic-opportunity $G_{H}\left(x ; 0, b_{c}^{*}\right)$ is

$$
\begin{aligned}
F_{L}(x)= & \left(\eta_{c}-\eta_{p} \frac{\gamma}{\gamma+\delta}\right)\left(\frac{s_{\gamma}}{s_{\gamma}-r_{\gamma}} \frac{e^{r_{\gamma}\left(x-b_{c}^{*}\right)}}{r_{\gamma}}+\frac{r_{\gamma}}{r_{\gamma}-s_{\gamma}} \frac{e^{s_{\gamma}\left(x-b_{c}^{*}\right)}}{s_{\gamma}}\right) \\
& +\frac{\eta_{p} \gamma}{\gamma+\delta}\left(x+\frac{\mu}{\gamma+\delta}\right), \quad \text { for } x \in\left[0, b_{c}^{*}\right) ; \\
F_{U}(x)= & \eta_{c}\left(x-b_{c}^{*}\right)+G_{M}\left(b_{c}^{*}\right), \quad \text { for } x \in\left[b_{c}^{*}, \infty\right),
\end{aligned}
$$

where the continuous barrier $b_{c}^{*}$ is the solution of the following equation:

$$
\left(\eta_{c}-\eta_{p} \frac{\gamma}{\gamma+\delta}\right)\left(\frac{s_{\gamma}}{s_{\gamma}-r_{\gamma}} \frac{e^{-r_{\gamma} b_{c}^{*}}}{r_{\gamma}}+\frac{r_{\gamma}}{r_{\gamma}-s_{\gamma}} \frac{e^{-s_{\gamma} b_{c}^{*}}}{s_{\gamma}}\right)+\frac{\eta_{p} \gamma}{\gamma+\delta} \frac{\mu}{\gamma+\delta}=0 .
$$


TABLE 1

OPTIMAL DIVIDEND STRATEGY UNDER DIFFERENT COMBINATIONS OF TRANSACTION COSTS.

\begin{tabular}{|c|c|c|c|}
\hline & $\begin{array}{l}\text { Positive drift }(\mu>0) \\
\text { and Insufficient- } \\
\text { prospect condition } \\
(3.34) \text { does not hold }\end{array}$ & $\begin{array}{l}\text { Positive drift }(\mu>0) \text { and } \\
\text { Insufficient-prospect } \\
\text { condition }(3.34) \text { holds }\end{array}$ & $\begin{array}{l}\text { Non-positive drift } \\
(\mu \leq 0)\end{array}$ \\
\hline $\begin{array}{l}\eta_{c} \in\left[\eta_{p}, 1\right) \\
\quad \text { Section } 4.5\end{array}$ & $\begin{array}{l}\text { Pure continuous } \\
\quad \text { barrier }\left({ }_{0} b_{c}^{*}>0\right)\end{array}$ & Not applicable & $\begin{array}{l}\text { Pure continuous } \\
\text { barrier with } \\
\text { immediate } \\
\text { liquidation } \\
\left({ }_{0} b_{c}^{*}=0\right)\end{array}$ \\
\hline $\begin{array}{l}\eta_{c} \in\left(\eta_{p} \frac{\gamma}{\gamma+\delta}, \eta_{p}\right) \\
\quad \text { Section } 4.3\end{array}$ & $\begin{array}{l}\text { Hybrid barrier } \\
\qquad\left(0<b_{p}^{*}<b_{c}^{*}<\infty\right)\end{array}$ & $\begin{array}{l}\text { Hybrid barrier with } \\
\text { liquidation-at-first- } \\
\text { periodic-opportunity } \\
\left(0=b_{p}^{*}<b_{c}^{*}<\infty\right)\end{array}$ & $\begin{array}{l}\text { Hybrid barrier with } \\
\text { immediate } \\
\text { liquidation } \\
\left(b_{p}^{*}=b_{c}^{*}=0\right)\end{array}$ \\
\hline $\begin{array}{l}\eta_{c} \in\left[0, \eta_{p} \frac{\gamma}{\gamma+\delta}\right] \\
\text { Section } 4.4\end{array}$ & $\begin{array}{l}\text { Pure periodic barrier } \\
\qquad\left({ }_{0} b_{p}^{*}>0\right)\end{array}$ & \multicolumn{2}{|c|}{$\begin{array}{l}\text { Pure periodic barrier with liquidation-at-first- } \\
\text { periodic-opportunity }\left({ }_{0} b_{p}^{*}=0\right)\end{array}$} \\
\hline
\end{tabular}

\section{THE INTERPLAY BETWEEN OPTIMAL PERIODIC AND CONTINUOUS DIVIDEND STRATEGIES}

In this section, we analyse the interaction between periodic and continuous dividend strategies under varying levels of proportional transaction costs. Our findings are summarised in Section 4.1 and Table 1. In order to prove those findings, a verification lemma is developed and proved in Section 4.2. In subsequent sections, we use the verification lemma to verify the following statements:

$$
\begin{aligned}
\eta_{c} \in\left[0, \eta_{p} \frac{\gamma}{\gamma+\delta}\right] & \Longleftrightarrow \text { A pure periodic barrier strategy is optimal; } \\
\eta_{c} \in\left(\eta_{p} \frac{\gamma}{\gamma+\delta}, \eta_{p}\right) & \Longleftrightarrow \text { A hybrid barrier strategy is optimal; } \\
\eta_{c} \in\left[\eta_{p}, 1\right] & \Longleftrightarrow \text { A pure continuous barrier strategy dominates. }
\end{aligned}
$$

\subsection{Summary of the findings}

Depending on the levels of profitability of the company (the surplus drift), and the respective levels of transaction costs on periodic and continuous dividends, different combinations of the continuous and periodic strategies will be optimal. These cases are summarised in Table 1; refer to Section 2.4 for the terminology.

In the first row, a pure continuous barrier strategy always dominates. When the net gain per dollar of periodic dividends is lower than that of continuous dividends - if $\eta_{c} \in\left[\eta_{p}, 1\right]$ - then the pure continuous barrier strategies are 
optimal in the set of hybrid strategies $\mathcal{D}_{H}$. The drift of the surplus process further determines whether immediate liquidation is optimal, i.e.,

$$
\begin{aligned}
& \mu>0 \Longleftrightarrow{ }_{0} b_{c}^{*}>0 ; \\
& \mu \leq 0 \Longleftrightarrow{ }_{0} b_{c}^{*}=0 .
\end{aligned}
$$

If the business is profitable (positive drift), then it is worthwhile to keep an investment in the company and receive dividends when the surplus reaches some strictly positive level $\left({ }_{0} b_{c}^{*}>0\right)$. On the other hand, if the business is not profitable $(\mu \leq 0)$, then the optimal strategy is to liquidate the initial surplus at time zero (see, for example, Avanzi et al., 2011).

In the second row, a hybrid barrier dividend strategy will be optimal. As the transaction costs on continuous dividends increase ( $\eta_{c}$ decreases) such that $\eta_{c} \in\left(\eta_{p} \frac{\gamma}{\gamma+\delta}, \eta_{p}\right)$, we have

$$
\begin{array}{ll}
G_{H}\left(b_{p}^{*} ; b_{p}^{*}, b_{c}^{*}\right)>0 & \Longleftrightarrow 0<b_{p}^{*}<b_{c}^{*}<\infty ; \\
\mu>0 \text { and } G_{H}\left(b_{p}^{*} ; b_{p}^{*}, b_{c}^{*}\right) \leq 0 & \Longleftrightarrow 0=b_{p}^{*}<b_{c}^{*}<\infty ; \\
\mu \leq 0 & \Longleftrightarrow b_{p}^{*}=b_{c}^{*}=0 .
\end{array}
$$

Under good prospects $\left(G_{H}\left(b_{p}^{*} ; b_{p}^{*}, b_{c}^{*}\right)>0\right)$, both hybrid periodic and continuous barriers are positive. As the prospects become insufficient (for example, low dividend frequencies) while the business still remains profitable in terms of expected value $(\mu>0)$, it may still be worthwhile to distribute continuous dividends. If periodic dividend decision times are relatively infrequent and periodic transaction costs are relatively low, one should liquidate the surplus at the first periodic dividend opportunity to take advantage of the low transaction costs (second column). In the case when the business is not profitable at all $(\mu \leq 0)$, the optimal strategy is to liquidate the initial surplus immediately (third column).

In the bottom row, periodic dividends are always preferable to continuous dividends. There, the transaction costs on continuous dividends are so high $\left(\eta_{c}\right.$ further decreases) that the net gain on continuous dividends is less than or equal the expected net gain on periodic dividends: $\eta_{c} \in\left[0, \eta_{p} \frac{\gamma}{\gamma+\delta}\right]$. In this case,

$$
\begin{aligned}
& G_{P}\left({ }_{0} b_{p}^{*} ;{ }_{0} b_{p}^{*}\right)>0 \Longleftrightarrow{ }_{0} b_{p}^{*}>0 ; \\
& G_{P}\left({ }_{0} b_{p}^{*} ;{ }_{0} b_{p}^{*}\right) \leq 0 \Longleftrightarrow{ }_{0} b_{p}^{*}=0 .
\end{aligned}
$$

Under good prospects $\left(G_{P}\left({ }_{0} b_{p}^{*} ;{ }_{0} b_{p}^{*}\right)>0\right)$, the optimal periodic barrier is strictly positive. Under insufficient prospects $\left(G_{P}\left({ }_{0} b_{p}^{*} ;{ }_{0} b_{p}^{*}\right) \leq 0\right)$, the optimal periodic strategy is to liquidate the surplus at the first periodic dividend opportunity. Note that while the latter will always happen when $\mu \leq 0$, it may also happen even if $\mu>0$. This behaviour was discussed in Avanzi et al. (2014). 


\subsection{Verification lemma}

Lemma 4.1. Suppose that we follow a hybrid dividend strategy $\Theta_{H}$. For a nonnegative function $H(x)$ that is twice continuously differentiable except at countable many points and that satisfies

1. $\gamma \max _{0 \leq l \leq x}\left\{\eta_{p} l+H(x-l)-H(x)\right\}+(\mathcal{A}-\delta) H(x) \leq 0$, with $H(0)=0$,

2. $\max \left(\eta_{c}, \eta_{p} \frac{\gamma}{\gamma+\delta}\right) \leq H^{\prime}(x)<\infty$,

3. $H^{\prime \prime}(x) \leq 0$,

for $x \geq 0$, where $(\mathcal{A}-\delta) H(x)=\frac{\sigma^{2}}{2} H^{\prime \prime}(x)+\mu H^{\prime}(x)-\delta H(x)$, then it holds that

$$
H(x) \geq V(x), \quad x \geq 0,
$$

where $V(x)$ is defined in (2.8).

Furthermore, when the optimal hybrid strategies are applied, if there exists a point $b_{c}^{*}<\infty$ such that

4. $\gamma \max _{0 \leq l \leq x}\left\{\eta_{p} l+H(x-l)-H(x)\right\}+(\mathcal{A}-\delta) H(x)=0$ and $H^{\prime}(x)>\eta_{c}$ for $x \in\left[0, b_{c}^{*}\right)$,

5. $(\mathcal{A}-\delta) H(x)<0$ and $H(x)=\eta_{c}\left(x-b^{*}\right)+H\left(b^{*}\right)$ for $x \in\left[b_{c}^{*}, \infty\right)$,

and if there exists $b_{p}^{*} \in\left[0, b_{c}^{*}\right)$ such that $H^{\prime}\left(b_{p}^{*}\right)=\eta_{p}$, then the optimal hybrid strategy is in the form

$$
D_{c}^{*}(t)=L_{X}^{b_{c}^{*}}(t) \quad \text { and } \quad D_{p}^{*}(t)=\sum_{i=1}^{N_{y}(t)}\left(X(t)-b_{p}^{*}\right)^{+}, \quad x \geq 0 .
$$

Otherwise, the optimal hybrid strategy is in the form of

$$
D_{c}^{*}(t)=L_{X}^{b_{c}^{*}}(t) \quad \text { and } \quad D_{p}^{*}(t)=0, \quad x \geq 0,
$$

where $L_{X}^{b_{c}^{*}}(t)=\int_{0}^{t} \mathbf{1}_{\left\{X(s)=b_{c}^{*}\right\}} d L_{X}^{b_{c}^{*}}(s)$ is a local time of the process $X$ at the barrier $b_{c}^{*}$.

If such a point $b_{c}^{*}$ does not exist and $H(x)$ satisfies

6. $\gamma \max _{0 \leq l \leq x}\left\{\eta_{p} l+H(x-l)-H(x)\right\}+(\mathcal{A}-\delta) H(x)=0$ for $x \in[0, \infty)$, then the optimal hybrid strategy is in the form

$$
D_{c}^{*}(t)=0 \quad \text { and } \quad D_{p}^{*}(t)=\sum_{i=1}^{N_{y}(t)}\left(X(t)-b_{p}^{*}\right)^{+}, \quad x \geq 0 .
$$

In all three cases described in (4.8)-(4.10), we have

$$
H(x)=V(x), \quad x \geq 0 .
$$


Proof 1. Using Itô formula for jump diffusion processes on $\left\{e^{-\delta(t \wedge \tau)} H(X(t \wedge\right.$ $\tau))\}$, we have

$$
\begin{aligned}
e^{-\delta(t \wedge \tau)} H(X(t \wedge \tau)) & =H(x)-\int_{0}^{t \wedge \tau} \delta e^{-\delta s} H(X(s)) d s \\
& +\int_{0}^{t \wedge \tau} e^{-\delta s} H^{\prime}(X(s)) d X^{(c)}(s) \\
& +\frac{\sigma^{2}}{2} \int_{0}^{t \wedge \tau} e^{-\delta s} H^{\prime \prime}(X(s)) d s+\sum_{s \leq t \wedge \tau}^{\Delta X(s) \neq 0} e^{-\delta s}[H(X(s-) \\
& +\Delta X(s))-H(X(s-))],
\end{aligned}
$$

where $X^{(c)}(t)$ is the continuous component of $X(t)$ and $\tau$ is the time of ruin. Note that $X^{(c)}(t)$ consists of the original surplus process and the continuous dividend process,

$$
d X^{(c)}(t)=\mu d t+\sigma d W(t)-d D_{c}(t),
$$

where $D_{c}(t)$ is the aggregate continuous dividend process before transaction costs. As $X(t)$ only jumps when a periodic dividend is issued, we can express the sum in (4.12) as a stochastic integral with respect to the Poisson process $\left\{N_{\gamma}(t)\right\}$,

$$
\begin{aligned}
& \sum_{s \leq t \wedge \tau}^{\Delta X(s) \neq 0} e^{-\delta s}[H(X(s-)+\Delta X(s))-H(X(s-))] \\
& =\int_{0}^{t \wedge \tau} e^{-\delta s}\left[\eta_{p} \vartheta_{s}+H\left(X(s-)-\vartheta_{s}\right)-H(X(s-))\right] d N_{\gamma}(s) \\
& \quad-\int_{0}^{t \wedge \tau} \eta_{p} e^{-\delta s} \vartheta_{s} d N_{\gamma}(s) .
\end{aligned}
$$

Next, we substitute (4.13) and (4.14) into (4.12) and after some algebraic simplifications, we have

$$
\begin{aligned}
& e^{-\delta(t \wedge \tau)} H(X(t \wedge \tau))=H(x) \\
& \quad+\int_{0}^{t \wedge \tau} e^{-\delta s}\left\{\gamma\left[\eta_{p} \vartheta_{s}+H\left(X(s-)-\vartheta_{s}\right)-H(X(s-))\right]+\frac{\sigma^{2}}{2} H^{\prime \prime}(x)\right. \\
& \left.\quad+\mu H^{\prime}(x)-\delta H(x)\right\} d s \\
& \quad+\int_{0}^{t \wedge \tau} \sigma e^{-\delta s} H^{\prime}(X(s)) d W(s)
\end{aligned}
$$




$$
\begin{aligned}
& +\int_{0}^{t \wedge \tau} e^{-\delta s}\left[\eta_{c}-H^{\prime}(X(s))\right] d D_{c}(s) \\
& +\int_{0}^{t \wedge \tau} e^{-\delta s}\left[\eta_{p} \vartheta_{s}+H\left(X(s-)-\vartheta_{s}\right)-H(X(s-))\right] d\left(N_{\gamma}(s)-\gamma s\right) \\
& -\int_{0}^{t \wedge \tau} \eta_{c} e^{-\delta s} d D_{c}(s)-\int_{0}^{t \wedge \tau} \eta_{p} e^{-\delta s} \vartheta_{s} d N_{\gamma}(s) .
\end{aligned}
$$

Now, since $H(x)$ is a concave function due to condition 3 , then $H^{\prime}(x)$ is bounded and the following stochastic integral

$$
\int_{0}^{t \wedge \tau} \sigma e^{-\delta s} H^{\prime}(X(s)) d W(s)
$$

is a uniformly integrable martingale and therefore the expectation of (4.16) is zero.

Second, we have $\eta_{c}-H^{\prime}(X(s)) \leq 0$ from condition 2 and since $D_{c}(t)$ is non-negative, so we have

$$
\int_{0}^{t \wedge \tau} e^{-\delta s}\left[\eta_{c}-H^{\prime}(X(s))\right] d D_{c}(s) \leq 0 .
$$

and therefore the expectation of (4.17) is less than zero.

Lastly, we show that (4.18) is a uniformly integrable martingale. Since (4.18) is a stochastic integral with respect to a compensated Poisson process $N_{\gamma}(t)$, so it suffices to show that

$$
\mathbb{E}^{x}\left[\int_{0}^{t \wedge \tau}\left(e^{-\delta s}\left[\eta_{p} \vartheta_{s}+H\left(X(s-)-\vartheta_{s}\right)-H(X(s-))\right]\right)^{2} d s\right]<\infty .
$$

Since $H$ is an increasing function from condition 2, we have

$$
\eta_{p} \vartheta_{s}+H\left(X(s-)-\vartheta_{s}\right)-H(X(s-)) \leq \eta_{p} \vartheta_{s} \leq X(s) .
$$

Next, since the modified surplus process is always less than or equal to the original surplus process, we have $X(s) \leq U(s)=x+\mu s+\sigma W(s)$ and this gives

$$
\begin{aligned}
& \sup _{t \geq 0} \mathbb{E}^{x}\left[\int_{0}^{t}\left(e^{-\delta s}\left[\eta_{p} \vartheta_{s}+H\left(X(s-)-\vartheta_{s}\right)-H(X(s-))\right]\right)^{2} d s\right] \\
& \quad \leq \sup _{t \geq 0} \mathbb{E}^{x}\left[\int_{0}^{t}\left(e^{-\delta s} U(s)\right)^{2} d s\right] .
\end{aligned}
$$

Since the process $\left\{e^{-\delta s} U(s)\right\}$ has regular sample path (see Definition 8.1, Klebaner, 2005), we can apply Fubini's theorem (see Theorem 2.39, Klebaner, 2005) 
to interchange the expectation and integration signs,

$$
\mathbb{E}^{x}\left[\int_{0}^{t} e^{-2 \delta s} U(s)^{2} d s\right]=\int_{0}^{t} e^{-2 \delta s} \mathbb{E}^{x}\left[U(s)^{2}\right] d s .
$$

Since $U(s) \sim N\left(x+\mu s, \sigma^{2} s\right), \mathbb{E}^{x}\left[U(s)^{2}\right]=\sigma^{2} s+(x+\mu s)^{2}$ and we have

$$
\begin{aligned}
\int_{0}^{t} e^{-2 \delta s}\left(\sigma^{2} s+(x+\mu s)^{2}\right) d s \\
=x^{2} \int_{0}^{t} e^{-2 \delta s} d s+\left(\sigma^{2}+2 x \mu\right) \int_{0}^{t} s e^{-2 \delta s} d s+\mu^{2} \int_{0}^{t} s^{2} e^{-2 \delta s} d s \\
=x^{2} \frac{1-e^{-2 \delta t}}{2 \delta}+\left(\sigma^{2}+2 x \mu\right) \frac{1-(1+2 \delta t) e^{-2 \delta t}}{4 \delta^{2}} \\
\quad+\mu^{2} \frac{1-(1+2 \delta t(1+\delta t)) e^{-2 \delta t}}{4 \delta^{3}}
\end{aligned}
$$

Hence, taking the supremum yields

$$
\sup _{t \geq 0} \mathbb{E}^{x}\left[\int_{0}^{t}\left(e^{-\delta s} U(s)\right)^{2} d s\right] \leq \frac{x^{2}}{2 \delta}+\frac{\sigma^{2}+2 x \mu}{4 \delta^{2}}+\frac{\mu^{2}}{4 \delta^{3}}<\infty .
$$

Combining (4.24) and (4.27) proves (4.22) and therefore (4.18) is a uniformly integrable martingale with zero expectation. Now, if we take expectation of the whole equation (4.19) and use the facts that (4.16) and (4.18) have zero expectations and (4.17) has a less-than-zero expectation, we have

$$
\begin{aligned}
& H(x) \geq \mathbb{E}^{x}\left[e^{-\delta(t \wedge \tau)} H(X(t \wedge \tau))\right] \\
& \quad+\mathbb{E}^{x}\left[\int_{0}^{t \wedge \tau} \eta_{c} e^{-\delta s} d D_{c}(s)+\int_{0}^{t \wedge \tau} \eta_{p} e^{-\delta s} \vartheta_{s} d N_{\gamma}(s)\right] .
\end{aligned}
$$

We now take the limit $t \rightarrow \infty$ on both sides of (4.28). We first use Fatou's lemma and have

$$
\lim _{t \rightarrow \infty} \mathbb{E}^{x}\left[e^{-\delta(t \wedge \tau)} H(X(t \wedge \tau))\right] \geq \mathbb{E}^{x}\left[\lim _{t \rightarrow \infty} e^{-\delta(t \wedge \tau)} H(X(t \wedge \tau))\right] .
$$

We can condition on the value of ruin time and have

$$
\begin{aligned}
& \lim _{t \rightarrow \infty} e^{-\delta(t \wedge \tau)} H(X(t \wedge \tau)) \\
& \quad=\lim _{t \rightarrow \infty} e^{-\delta(t \wedge \tau)} H(X(t \wedge \tau)) \mathbf{1}_{\{\tau<\infty\}}+\lim _{t \rightarrow \infty} e^{-\delta(t \wedge \tau)} H(X(t \wedge \tau)) \mathbf{1}_{\{\tau=\infty\}} \\
& =e^{-\delta(\tau)} H(X(\tau)) \mathbf{1}_{\{\tau<\infty\}}+\lim _{t \rightarrow \infty} e^{-\delta t} H(X(t)) \mathbf{1}_{\{\tau=\infty\}} .
\end{aligned}
$$


Since $H(X(\tau))=0$, we have

$$
\lim _{t \rightarrow \infty} \mathbb{E}^{x}\left[e^{-\delta(t \wedge \tau)} H(X(t \wedge \tau))\right] \geq 0
$$

We now consider the case when conditions 4-6 are satisfied. First, assume that there exists a point $b_{c}^{*}<\infty$ such that conditions 4 and 5 are satisfied and the optimal hybrid strategies are in the form of either (4.8) or (4.9). In either cases, the modified surplus process is bounded between $0 \leq X(t) \leq b_{c}^{*}$ for $t \geq 0$. Next, we substitute condition 4 into (4.15). Using the fact that (4.16) and (4.18) are uniformly integrable martingales, taking expectation of (4.15) yields

$$
\begin{aligned}
H(x)= & \mathbb{E}^{x}\left[e^{-\delta(t \wedge \tau)} H(X(t \wedge \tau))\right] \\
& -\mathbb{E}^{x}\left[\int_{0}^{t \wedge \tau} e^{-\delta s}\left[\eta_{c}-H^{\prime}(X(s))\right] d D_{c}^{*}(s)\right] \\
& +\mathbb{E}^{x}\left[\int_{0}^{t \wedge \tau} \eta_{c} e^{-\delta s} d D_{c}^{*}(s)+\int_{0}^{t \wedge \tau} \eta_{p} e^{-\delta s} d D_{p}^{*}(s)\right] .
\end{aligned}
$$

From the definition of $D_{c}^{*}(s)$ in (4.8) and (4.9), continuous dividends are only distributed when the surplus process reaches $b_{c}^{*}$, so we can write

$$
\begin{aligned}
\mathbb{E}^{x} & {\left[\int_{0}^{t \wedge \tau} e^{-\delta s}\left[\eta_{c}-H^{\prime}(X(s))\right] d D_{c}^{*}(s)\right] } \\
& =\mathbb{E}^{x}\left[\int_{0}^{t \wedge \tau} e^{-\delta s}\left[\eta_{c}-H^{\prime}(X(s))\right] \mathbf{1}_{\left\{X(s)=b_{c}^{*}\right\}} d D_{c}^{*}(s)\right] \\
& =\mathbb{E}^{x}\left[\int_{0}^{t \wedge \tau} e^{-\delta s}\left[\eta_{c}-\eta_{c}\right] \mathbf{1}_{\left\{X(s)=b_{c}^{*}\right\}} d D_{c}^{*}(s)\right]=0 .
\end{aligned}
$$

In (4.33), we use the fact that $H^{\prime}$ is a continuous and decreasing function from condition 3 of the verification lemma and have $H^{\prime}(X(s)) \mathbf{1}_{\left\{X(s)=b_{c}^{*}\right\}}=\eta_{c}$ for $s \geq 0$. Next, by conditioning on the value of $t$ relative to $\tau$ and using the fact that $X(t)$ is upper bounded by $b_{c}^{*}$, we have

$$
0 \leq e^{-\delta(t \wedge \tau)} H(X(t \wedge \tau))=e^{-\delta t} H(X(t)) \mathbf{1}_{\{t<\tau\}} \leq e^{-\delta t} H\left(b_{c}^{*}\right)
$$

Taking the limit of $t \rightarrow \infty$, we have $\lim _{t \rightarrow \infty} \mathbb{E}^{x}\left[e^{-\delta(t \wedge \tau)} H(X(t \wedge \tau))\right]=0$. Substituting this and (4.33) into (4.32) and using the monotone convergence 
theorem,

$$
\begin{aligned}
H(x) & =\lim _{t \rightarrow \infty} \mathbb{E}^{x}\left[\int_{0}^{t \wedge \tau} \eta_{c} e^{-\delta s} d D_{c}^{*}(s)+\int_{0}^{s \wedge \tau} \eta_{p} e^{-\delta s} d D_{p}^{*}(s)\right] \\
& =\mathbb{E}^{x}\left[\int_{0}^{\tau} \eta_{c} e^{-\delta s} d D_{c}^{*}(s)+\int_{0}^{\tau} \eta_{p} e^{-\delta s} d D_{p}^{*}(s)\right] \\
& =V(x) .
\end{aligned}
$$

Next, suppose that such a point $b_{c}^{*}<\infty$ does not exist and condition 6 is satisfied, the optimal hybrid strategies are in the form of (4.10). A similar proof can be obtained by removing the jump component in Section 3.4 in Avanzi et al. (2014) to show that the periodic barrier strategies are optimal in the set of hybrid strategies.

\subsection{On the global optimality of the hybrid barrier strategies}

In this section, we prove that if the net gain on continuous dividends $\left(\eta_{c}\right)$ falls strictly in the interval $\left(\eta_{p} \frac{\gamma}{\gamma+\delta}, \eta_{p}\right)$, then the optimal hybrid barrier strategies are optimal in the set of all hybrid strategies,

$$
\eta_{c} \in\left(\eta_{p} \frac{\gamma}{\gamma+\delta}, \eta_{p}\right) \Longrightarrow \text { A hybrid barrier strategy is optimal. }
$$

Suppose that the net gain on continuous dividends satisfies $\eta_{c} \in\left(\eta_{p} \frac{\gamma}{\gamma+\delta}, \eta_{p}\right)$. We need to show that the expected present value of dividends until ruin $G_{H}\left(x ; b_{p}^{*}, b_{c}^{*}\right)$ satisfies conditions $2-5$ in the verification lemma. First, we prove that $G_{H}\left(x ; b_{p}^{*}, b_{c}^{*}\right)$ satisfies conditions 2 and 3 by showing that it is an increasing and concave function - this is done in Appendix A.2.

Next, we show that $G_{H}\left(x ; b_{p}^{*}, b_{c}^{*}\right)$ satisfies condition 4. From (3.4) and (3.6), the function $G_{H}\left(x ; b_{p}^{*}, b_{c}^{*}\right)$ satisfies

$$
\begin{aligned}
& (\mathcal{A}-\delta) G_{H}\left(x ; b_{p}^{*}, b_{c}^{*}\right)=0 \quad x \in\left[0, b_{p}^{*}\right) ; \\
& \gamma\left[\eta_{p}\left(x-b_{p}^{*}\right)+G_{H}\left(b_{p}^{*} ; b_{p}^{*}, b_{c}^{*}\right)-G_{H}\left(x ; b_{p}^{*}, b_{c}^{*}\right)\right]+(\mathcal{A}-\delta) G_{H}\left(x ; b_{p}^{*}, b_{c}^{*}\right) \\
& \quad=0 \quad x \in\left[b_{p}^{*}, b_{c}^{*}\right) .
\end{aligned}
$$

Using Lemma 3.1 from Avanzi et al. (2014) and due to concavity of $G_{H}\left(x ; b_{p}^{*}, b_{c}^{*}\right)$ and $G_{H}^{\prime}\left(b_{p}^{*} ; b_{p}^{*}, b_{c}^{*}\right)=\eta_{p},(4.37)$ and (4.38) together attain the maximum of the following equation:

$$
\begin{gathered}
\gamma \max _{0 \leq l \leq x}\left[\eta_{p} l+G_{H}\left(x-l ; b_{p}^{*}, b_{c}^{*}\right)+G_{H}\left(x ; b_{p}^{*}, b_{c}^{*}\right)\right]+(\mathcal{A}-\delta) G_{H}\left(x ; b_{p}^{*}, b_{c}^{*}\right) \\
=0 \text { for } x \in\left[0, b_{c}^{*}\right) .
\end{gathered}
$$

Therefore, $G_{H}\left(x ; b_{p}^{*}, b_{c}^{*}\right)$ for $x \in\left[0, b_{c}^{*}\right)$ satisfies condition 4 in the verification lemma. 
Lastly, we show that $G_{H}\left(x ; b_{p}^{*}, b_{c}^{*}\right)$ satisfies the condition 5 in the verification lemma for $x \in\left[b_{c}^{*}, \infty\right)$. We substitute $G_{H}\left(x ; b_{p}^{*}, b_{c}^{*}\right)=\eta_{c}\left(x-b_{c}^{*}\right)+$ $G_{H}\left(b_{c}^{*} ; b_{p}^{*}, b_{c}^{*}\right)$ into the inequality in condition 5 ,

$$
(\mathcal{A}-\delta) G_{H}\left(x ; b_{p}^{*}, b_{c}^{*}\right)=\mu \eta_{c}-\delta G_{H}\left(x ; b_{p}^{*}, b_{c}^{*}\right)<\mu \eta_{c}-\delta G_{H}\left(b_{c}^{*} ; b_{p}^{*}, b_{c}^{*}\right) .
$$

We can substitute $x=b_{c}^{*}$ into (3.22) and have

$$
G_{H}\left(b_{c}^{*} ; b_{p}^{*}, b_{c}^{*}\right)=\eta_{c} \frac{\mu}{\gamma+\delta}+\frac{\gamma}{\gamma+\delta}\left(\eta_{p}\left(b_{c}^{*}-b_{p}^{*}\right)+G_{H}\left(b_{p}^{*} ; b_{p}^{*}, b_{c}^{*}\right)\right) .
$$

Since $G_{H}\left(x ; b_{p}^{*}, b_{c}^{*}\right)$ is increasing and concave, then by the Mean Value Theorem, we have $G_{H}\left(b_{c}^{*} ; b_{p}^{*}, b_{c}^{*}\right)<\eta_{p}\left(b_{c}^{*}-b_{p}^{*}\right)+G_{H}\left(b_{p}^{*} ; b_{p}^{*}, b_{c}^{*}\right)$. Substituting this inequality into (4.41) yields

$$
\eta_{c} \frac{\mu}{\gamma+\delta}+\frac{\gamma}{\gamma+\delta} G_{H}\left(b_{c}^{*} ; b_{p}^{*}, b_{c}^{*}\right)<G_{H}\left(b_{c}^{*} ; b_{p}^{*}, b_{c}^{*}\right) \Longleftrightarrow G_{H}\left(b_{c}^{*} ; b_{p}^{*}, b_{c}^{*}\right)>\eta_{c} \frac{\mu}{\delta} \text {. }
$$

Substituting (4.42) into (4.40) shows that $(\mathcal{A}-\delta) G_{H}\left(x ; b_{p}^{*}, b_{c}^{*}\right)<0$ for $x \in$ $\left[b_{c}^{*}, \infty\right)$, where $G_{H}\left(x ; b_{p}^{*}, b_{c}^{*}\right)=\eta_{c}\left(x-b_{c}^{*}\right)+G_{H}\left(b_{c}^{*} ; b_{p}^{*}, b_{c}^{*}\right)$. This shows that $G_{H}\left(x ; b_{p}^{*}, b_{c}^{*}\right)$ satisfies condition 5 and the proof is complete.

4.3.1. On the optimality of hybrid barrier strategies with liquidation-at-firstperiodic-opportunity. In this section, we show that if the insufficient-prospect condition is satisfied (i.e., $G_{H}\left(b_{p}^{*} ; b_{p}^{*}, b_{c}^{*}\right) \leq 0$ ), then the hybrid barrier strategy with liquidation-at-first-periodic-opportunity is optimal. Before we proceed, we present a useful Lemma, whose proof is included in Appendix A.5.

Lemma 4.2. The insufficient-prospect condition $G_{H}\left(b_{p}^{*} ; b_{c}^{*}, b_{p}^{*}\right) \leq 0$ is satisfied if and only if the function $F_{L}(x)$ derived in (3.41) satisfies $F_{L}^{\prime}(0) \leq \eta_{p}$.

Now, suppose that the insufficient-prospect condition is satisfied, then it remains to verify that the function $G_{H}\left(x ; 0, b_{c}^{*}\right)$ satisfies conditions $2-5$ in the verification lemma. In Section A.4, we demonstrate that $G_{H}\left(x ; 0, b_{c}^{*}\right)$ is an increasing and concave function, which shows that conditions 2 and 3 are satisfied. Next, from (3.36) and (3.37), the function $G_{H}\left(x ; 0, b_{c}^{*}\right)$ satisfies

$$
\begin{aligned}
\gamma\left[\eta_{p} x-F_{L}(x)\right]+(\mathcal{A}-\delta) F_{L}(x)=0 & \text { for } x \in\left[0, b_{c}^{*}\right), \\
F_{U}(x)=\eta_{c}\left(x-b_{c}^{*}\right)+F_{L}\left(b_{c}^{*}\right) & \text { for } x \in\left[b_{c}^{*}, \infty\right) .
\end{aligned}
$$

To verify conditions 4 and 5 , it remains to show that for $x \in\left[0, b_{c}^{*}\right)$,

$$
\gamma \max _{0 \leq l \leq x}\left\{\eta_{p} l+F_{L}(x-l)-F_{L}(x)\right\}=\gamma\left[\eta_{p} x-F_{L}(x)\right] .
$$

Since the insufficient-prospect condition is satisfied $\left(G_{H}\left(b_{p}^{*} ; b_{p}^{*}, b_{c}^{*}\right) \leq 0\right)$, by Lemma 4.2, it implies that $F_{L}^{\prime}(0) \leq \eta_{p}$. Due to concavity of $F_{L}(x)$, we have 
$F_{L}^{\prime}(x) \leq \eta_{p}$ for $x \in\left[0, b_{c}^{*}\right)$ and we have

$$
\frac{d}{d l}\left(\eta_{p} l+F_{L}(x-l)-F_{L}(x)\right)=\eta_{p}-F_{L}^{\prime}(x-l) \geq 0, \quad \text { for } 0 \leq l \leq x .
$$

Then, $\max _{0 \leq l \leq x}\left\{\eta_{p} l+F_{L}(x-l)-F_{L}(x)\right\}$ has to occur at the endpoint $l=x$ and this gives (4.43) and the proof is complete.

\subsection{On the global optimality of the pure periodic barrier strategies}

In this section, we show that if the proportional transaction costs on continuous dividends are at a level such that $\eta_{c} \in\left[0, \eta_{p} \frac{\gamma}{\gamma+\delta}\right]$, then the optimal periodic barrier strategies are optimal in the set of hybrid strategies,

$$
\eta_{c} \in\left[0, \eta_{p} \frac{\gamma}{\gamma+\delta}\right] \Longrightarrow \text { A pure periodic barrier strategy is optimal. }
$$

Now, assume that $\eta_{c}$ satisfies $\eta_{c} \in\left[0, \eta_{p} \frac{\gamma}{\gamma+\delta}\right]$. We need to show that the function $G_{P}\left(x ;{ }_{0} b_{p}^{*}\right)$ in $(2.11)$ satisfies conditions 2,3 and 6 of the verification lemma. Details of this can be found in Sections 5.1 and 5.2 in Avanzi et al. (2014) by removing the jumps in the surplus process.

\subsection{On the global optimality of the pure continuous barrier strategies}

In this section, we show that if the proportional transaction costs on continuous dividends are at a level such that $\eta_{c} \in\left[\eta_{p}, 1\right]$, then the optimal continuous barrier strategies are optimal in the set of hybrid strategies,

$$
\eta_{c} \in\left[\eta_{p}, 1\right] \Longrightarrow \text { A pure continuous barrier strategy is optimal. }
$$

Now, suppose that the net gain of continuous dividends $\eta_{c}$ satisfies the inequality $\eta_{p} \leq \eta_{c} \leq 1$. We show that the expected present value of dividends of a continuous dividend strategy $G_{C}\left(x ;{ }_{0} b_{c}^{*}\right)$ in (2.9) satisfies conditions 2-5 in the verification lemma. It is well known that $G_{C}\left(x ;{ }_{0} b_{c}^{*}\right)$ satisfies conditions 2, 3 and 5 (see, e.g., Jeanblanc-Picqué and Shiryaev, 1995). It remains to show that $G_{C}\left(x ;{ }_{0} b_{c}^{*}\right)$ satisfies condition 4 . Since we know that $G_{C}^{\prime}\left(x ;{ }_{0} b_{c}^{*}\right) \geq \eta_{c}$ for $x \in\left[0, b_{c}^{*}\right)$ due to concavity of $G_{C}\left(x ;{ }_{0} b_{c}^{*}\right)$ and $\eta_{p} \leq \eta_{c}$, there is no $x \in\left[0, b_{c}^{*}\right)$ such that $G_{C}^{\prime}\left(x ;{ }_{0} b_{c}^{*}\right)=\eta_{p}$ and therefore

$$
\gamma \max _{0 \leq l \leq x}\left\{\eta_{p} l+G_{C}\left(x-l ;{ }_{0} b_{c}^{*}\right)-G_{C}\left(x ;{ }_{0} b_{c}^{*}\right)\right\}=0 \quad \text { for } \quad x \in\left[0, b_{c}^{*}\right) .
$$

Furthermore, since $G_{C}\left(x ;{ }_{0} b_{c}^{*}\right)$ for $x \in\left[0, b_{c}^{*}\right)$ is derived by solving $(\mathcal{A}-$ ס) $G_{C}\left(x ;{ }_{0} b_{c}^{*}\right)=0$, we have shown that condition 4 is satisfied and the proof is complete. 


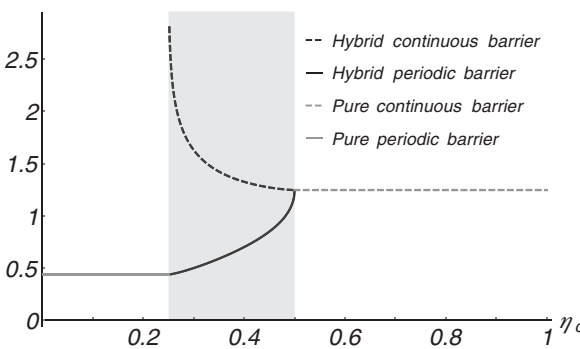

(a)

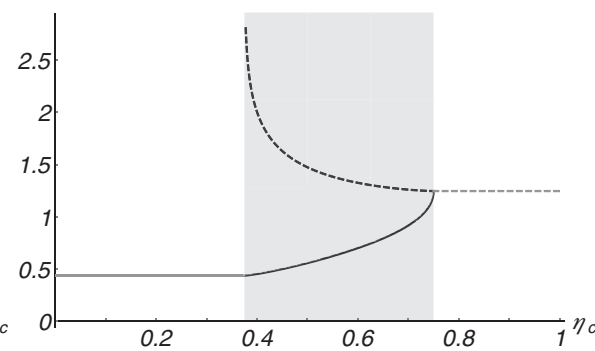

(b)

FIGURE 2: Continuous, hybrid and periodic barriers as functions of $\eta_{c} . \delta=0.5, \sigma=1, \gamma=0.5$. (a) $\eta_{p}=0.5 \Longrightarrow\left(\eta_{p} \frac{\gamma}{\gamma+\delta}, \eta_{p}\right)=(0.25,0.5)$. (b) $\eta_{p}=0.75 \Longrightarrow\left(\eta_{p} \frac{\gamma}{\gamma+\delta}, \eta_{p}\right)=(0.375,0.75)$.

\section{NUMERICAL ILLUSTRATIONS}

In Table 1 on page 18, we provided a summary of the optimal dividend strategies under different combinations of transaction costs $\left(\eta_{c}\right.$ and $\left.\eta_{p}\right)$, dividend frequency $(\gamma)$ and force of interest $(\delta)$. In this section, we illustrate our findings. In particular, we consider transitions between different optimal strategy types.

\subsection{The interface between pure continuous and periodic strategies via hybrid strategies}

We start by offering a pictural representation of our paper, that is, of the interface between the optimal (pure) periodic and continuous strategies, when transaction costs are included.

Figure 2 displays the pure continuous and periodic barriers $\left({ }_{0} b_{c}^{*}\right.$ and $\left.{ }_{0} b_{p}^{*}\right)$ with the grey dashed and solid lines using equations (2.10) and (2.12). The hybrid continuous and periodic barriers $\left(b_{c}^{*}\right.$ and $\left.b_{p}^{*}\right)$ are shown using the black dashed and solid lines, respectively. Here, we change the level of $\eta_{c}$ from 0 to 1 and fix $\eta_{p}$ to be 0.5 and 0.75 on the left-hand and right-hand sides, respectively. Note that the barriers provided in the shaded area (the hybrid barriers in solid black) are calculated using results from this paper, whereas the grey barrier is calculated thanks to formulas in the papers cited in Section 2.4.

The grey region corresponds to the cases where a hybrid strategy is optimal, that is, when $\eta_{c} \in\left(\eta_{p} \frac{\gamma}{\gamma+\delta}, \eta_{p}\right)$. On its left, the pure periodic barrier strategy is optimal. On its right, the pure continuous barrier strategy is optimal. The right bound of this region is defined by $\eta_{p}$ : if $\eta_{c}$ is higher (that is, if dividends paid from a continuous strategy attract the lowest transaction costs), then a pure continuous strategy is obviously optimal. The left bound depends on the ratio between $\gamma$ (the intensity to pay dividends periodically) and $\delta$ (the indicator of time impatience in the model). The higher that ratio is, the closer the left bound is to the right bound, which makes sense: if dividends can be paid relatively often, and at lower transaction costs, then the attractivity of dividends paid from 
the continuous barrier decreases and a pure periodic barrier strategy is more likely to be optimal.

In the shaded area, as $\eta_{c}$ increases, the net gain on hybrid continuous dividends improves and they become a marginally more attractive alternative to the hybrid periodic dividends. This is represented by the diminishing gap of $b_{c}^{*}-b_{p}^{*}$. The decreasing $b_{c}^{*}$ allows for an earlier access to continuous dividends, while the increasing $b_{p}^{*}$ delays the distribution of periodic dividends. It is interesting to note that this gap is a key quantity when solving for the expected present value of dividends of a hybrid strategy; recall equations (3.23) and (3.24).

\subsection{Dominance of the hybrid barrier strategy}

In Section 5.1, we illustrated how the hybrid barrier strategies are connected to the pure continuous and periodic strategies. As explained earlier, the hybrid barrier strategies are optimal in the grey regions of Figure 2; we illustrate this dominance in this section.

We start by examining the dominance of the hybrid barrier strategies from the point of view of transaction costs $\eta_{c}$ (using the same angle as in Section 5.1). In Figure 3(a), we plot the expected present value of dividends $G_{P}\left(1 ;{ }_{0} b_{p}^{*}\right)$, $G_{C}\left(1 ; 0 b_{c}^{*}\right)$ and $G_{H}\left(1 ; b_{p}^{*}, b_{c}^{*}\right)$ as functions of $\eta_{c}$. First, note that $G_{P}\left(1 ;{ }_{0} b_{p}^{*}\right)$ in a pure periodic barrier strategy is unaffected by the change of $\eta_{c}$ (the flat grey solid line). In a pure continuous barrier strategy, $G_{C}\left(1 ;{ }_{0} b_{c}^{*}\right)$ is a linear function that increases with $\eta_{c}$ (the grey dashed line). Both $G_{P}\left(1 ;{ }_{0} b_{p}^{*}\right)$ and $G_{C}\left(1 ;{ }_{0} b_{c}^{*}\right)$ are calculated using formulas reviewed in Section 2.4. Lastly, we plot $G_{H}\left(1 ; b_{p}^{*}, b_{c}^{*}\right)$ in its respective domain of $\eta_{c}$ (the black solid line in the shaded region), which is similar to Figure 2. As $\eta_{c}$ approaches the boundaries of its domain (the upper and lower bounds of the shaded region), the dominance of the hybrid barrier strategies vanishes and the pure continuous or periodic strategies become optimal. This is similar to the behaviour explained in previous Section 5.1. Importantly, this graph shows that the optimality question does not boil down to choosing between a pure periodic or a pure continuous strategy, but that a combination of both is actually optimal in certain cases.

Next, we illustrate the dominance of the hybrid barrier strategies from the viewpoint of dividend frequency $(\gamma)$. From Section 4.3, we know that for given levels of transaction costs $\left(\eta_{c}\right.$ and $\left.\eta_{p}\right)$, the frequency of hybrid periodic dividends $(\gamma)$ has a natural domain,

$$
\eta_{p} \frac{\gamma}{\gamma+\delta}<\eta_{c} \Longrightarrow \gamma \in\left(0, \delta \frac{\eta_{c}}{\eta_{p}-\eta_{c}}\right) \text {. }
$$

This redefines the left bound of the shaded areas as a function of $\gamma$ under a hybrid barrier strategy.

In Figure 3(b), we plot the expected present value of dividends $G_{P}\left(1 ;{ }_{0} b_{p}^{*}\right)$, $G_{C}\left(1 ;{ }_{0} b_{c}^{*}\right)$ and $G_{H}\left(1 ; b_{p}^{*}, b_{c}^{*}\right)$ as functions of $\gamma$. One first notice that $G_{C}\left(1 ;{ }_{0} b_{c}^{*}\right)$ in a pure continuous strategy is unaffected by the increasing $\gamma$ (the flat dashed 


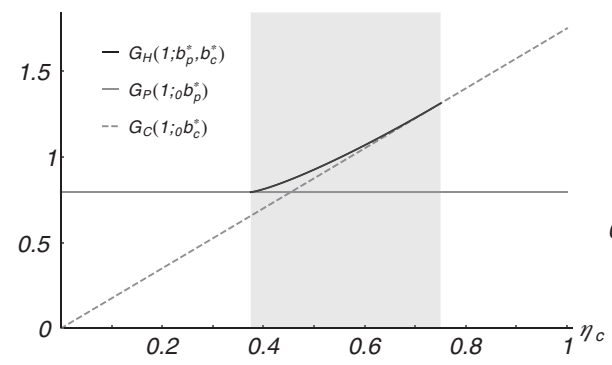

(a)

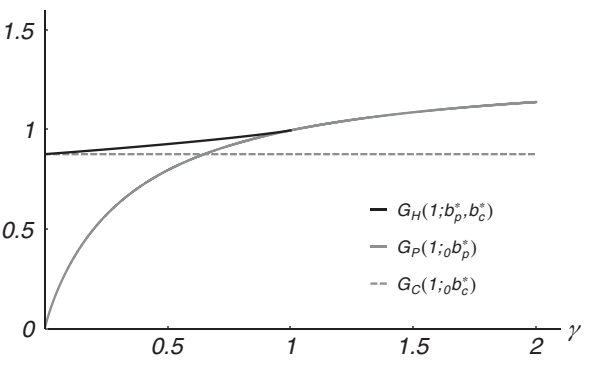

(b)

FIGURE 3: Expected present value of dividends $G_{H}\left(1 ; b_{p}^{*}, b_{c}^{*}\right), G_{P}\left(1 ;{ }_{0} b_{p}^{*}\right)$ and $G_{C}\left(1 ;{ }_{0} b_{c}^{*}\right)$. (a) $\gamma=0.5, \eta_{p}=0.75 \Longrightarrow\left(\eta_{p} \frac{\gamma}{\gamma+\delta}, \eta_{p}\right)=(0.375,0.75)$. (b) $\eta_{p}=0.75, \eta_{c}=0.5 \Longrightarrow\left(0, \delta \frac{\eta_{c}}{\eta_{p}-\eta_{c}}\right)=(0,1)$.

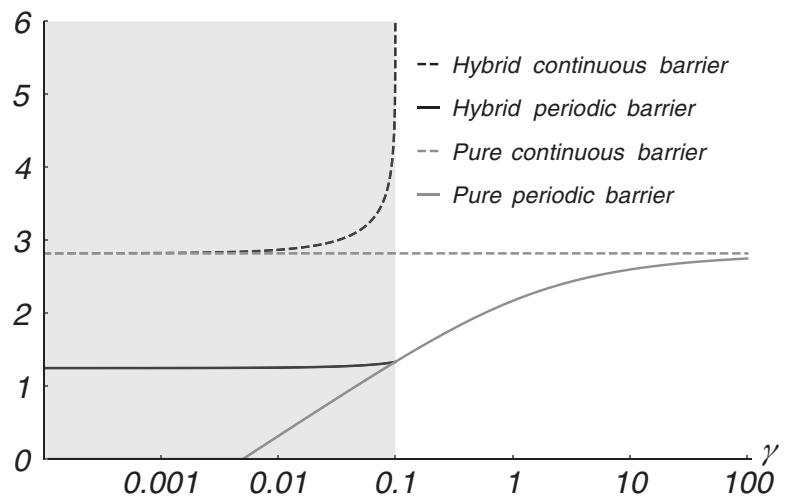

FIGURE 4: Continuous, hybrid and periodic barriers as functions of $\gamma$. $\delta=0.1, \sigma=1, \mu=1, \eta_{c}=0.5, \eta_{p}=1$.

grey line) and $G_{P}\left(1 ;{ }_{0} b_{p}^{*}\right)$ in a pure periodic strategy increases as dividends are distributed more frequently (the solid grey line). Last, we plot $G_{H}\left(1 ; b_{p}^{*}, b_{c}^{*}\right)$ (the black solid line), which lies above both $G_{P}\left(1 ;{ }_{0} b_{p}^{*}\right)$ and $G_{C}\left(1 ;{ }_{0} b_{c}^{*}\right)$. One can see that the premium obtained by the opportunity to pay continuously or periodically - the difference between black and grey lines, for given $\gamma$ - reduces as $\gamma$ increases to eventually vanish where black and solid grey line intersect. Beyond that point, a pure periodic strategy is optimal.

\subsection{Continuous, hybrid and periodic barriers as functions of $\gamma$}

In this section, we keep the angle defined in the previous section and re-plot Figure 2, but as a function of $\gamma$. Since $\gamma$ is considered here, the right-hand side (sic, the orientation of the graph is flipped around horizontally) area of Figure 2 is not plotted, but corresponds to the limit on the $y$-axis where pure and hybrid continuous barriers meet. 


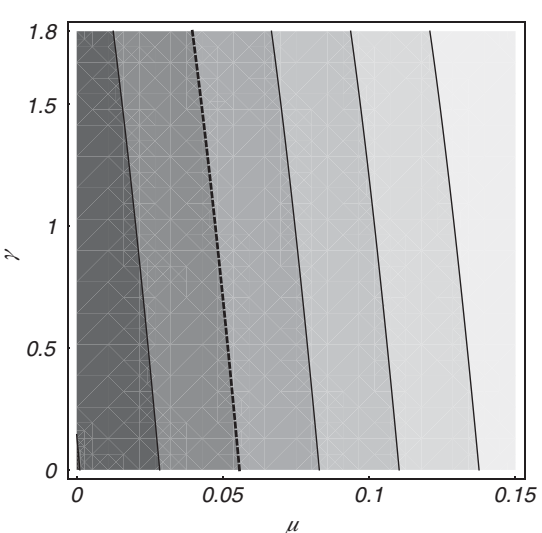

(a)

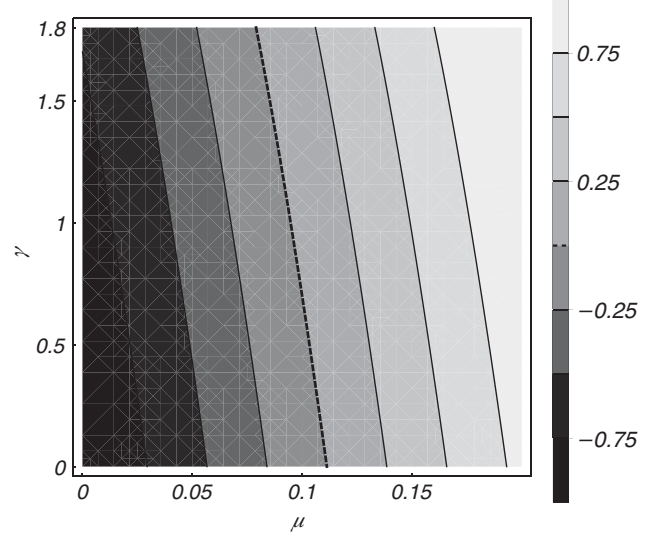

(b)

FIGURE 5: Contour plots of $G_{H}\left(b_{p}^{*} ; b_{p}^{*}, b_{c}^{*}\right)$ as a function of $\mu$ and $\gamma$. (a) $\delta=0.1, \eta_{c}=0.9, \eta_{p}=0.95, \sigma=0.75$. (b) $\delta=0.1, \eta_{c}=0.9, \eta_{p}=0.95, \sigma=1.5$.

In Figure 4, we plot the hybrid periodic and continuous barriers in an optimal hybrid strategy ( $b_{p}^{*}$ and $b_{c}^{*}$, solid and dashed black lines), the periodic barrier in an optimal pure periodic strategy $\left({ }_{0} b_{p}^{*}\right.$, grey solid line) and the continuous barrier in an optimal pure continuous strategy ( ${ }_{0} b_{c}^{*}$, grey dashed line) in the same graph as we change dividend frequency $(\gamma)$.

First, note that the pure continuous barrier ${ }_{0} b_{c}^{*}$ is constant with respect to $\gamma$ (unsurprisingly). On the other hand, the pure periodic barrier ${ }_{0} b_{p}^{*}$ starts at zero for small $\gamma$ (due to liquidating-at-first-periodic-opportunity). As $\gamma$ increases, the pure periodic barrier increases and approaches the pure continuous barrier (i.e., $\left.\lim _{\gamma \rightarrow \infty} b_{p}^{*}={ }_{0} b_{c}^{*}\right)$. Next, we note that the natural domain of $\gamma$ in an optimal hybrid barrier strategy is $(0,0.1)$ according to $(5.1)$, which is represented by the shaded region. As $\gamma \rightarrow \delta \frac{\eta_{c}}{\eta_{p}-\eta_{c}}$, the hybrid continuous barrier approaches infinity $\left(b_{c}^{*} \rightarrow \infty\right)$ and the hybrid periodic barrier approaches the pure periodic barrier $\left(b_{p}^{*} \rightarrow{ }_{0} b_{p}^{*}\right)$.

\subsection{Situations leading to the insufficient-prospect condition}

In Section 4.3.1, we showed that under the insufficient-prospect condition $\left(G_{H}\left(b_{p}^{*} ; b_{p}^{*}, b_{c}^{*}\right) \leq 0\right)$ and with positive drift, the optimal strategy is hybrid with liquidation-at-first-periodic-opportunity $\left(0=b_{p}^{*}<b_{c}^{*}<\infty\right)$. In Figure 2, this would translate into a solid black line touching the $x$-axis for some range of $\eta_{c}$ on the left of the shaded area. In this section, we illustrate different combinations of parameters that lead to the insufficient-prospect condition.

In Figures 5(a) and (b), we display two contour plots of $G_{H}\left(b_{p}^{*} ; b_{p}^{*}, b_{c}^{*}\right)$ against the drift $(\mu)$ and the frequency of periodic dividends $(\gamma)$ using the explicit formula in (3.28). Because of (5.1), Figure 5 focusses on the range $\gamma<1.8$. 
Both graphs are separated into two regions by a black dashed line, which represent the frontier that consists all pairs of $(\mu, \gamma)$ that produce $G_{H}\left(b_{p}^{*} ; b_{p}^{*}, b_{c}^{*}\right)=0$. If the parameters $\mu$ and $\gamma$ fall into the region to the left of the dashed line (i.e., satisfying the insufficient-prospect condition), the optimal hybrid barrier strategy is to liquidate at the first periodic dividend time $\left(b_{p}^{*}=0\right)$, but to still pay continuous dividends according to a barrier strategy in the meantime $\left(0<b_{c}^{*}<\infty\right)$.

In a pure periodic framework, the liquidation-at-first-periodic-opportunity strategy becomes optimal when the frequency of dividend payments becomes too low, regardless of the drift of the surplus process; see also Section 4.4 in Avanzi et al. (2014). This is not true in a hybrid barrier strategy. Suppose that the drift of the surplus $(\mu)$ is high enough such that it falls in the region to the right of the dashed lines in Figure 5, then $G_{H}\left(b_{p}^{*} ; b_{p}^{*}, b_{c}^{*}\right)$ is strictly positive for all possible values of $\gamma$ and the liquidation-at-first-periodic-opportunity strategy is never optimal (the dashed line intersects the $x$-axis at $\gamma=0$, it does not approach it). Despite low periodic dividend frequencies, one should still let the business carry on. This is because of the availability of dividend payments thanks to a hybrid continuous barrier; such an opportunity does not exist in a pure periodic strategy.

\section{CONCLUSION}

In this paper, we teased out the relationship between so-called continuous and periodic dividend strategies, in a Brownian risk model. We obtained explicit expressions for the expected present value of dividends paid until ruin for different levels of transaction costs $\left(\eta_{c}\right.$ and $\left.\eta_{p}\right)$, and for different levels of expected profit $(\mu)$; see Table 1 . We formally determined which dividend strategy (or combination thereof) is optimal in all those cases. A key assumption is that periodic dividends (assumed to be of a regular nature) attract lower transaction costs than continuous dividends (to organise extra, immediate payments is more costly than to issue regular dividends).

This paper showed and illustrated, for the first time, how those two types of dividend strategies interact. Interestingly, they not only connect nicely, but it also turns out that a combination of both - referred to as a "hybrid" strategy - does constitute an optimal interface in some cases. Figure 2 illustrates this very nicely.

While proportional transaction costs form a reasonable approximation and are enough for drawing qualitative conclusions, in reality transaction costs have fixed and variable components. Furthermore, differences of costs between proportional and continuous dividends are likely to manifest themselves in that fixed component. Hence, fixed transaction costs would be a welcome addition to the model. Finally, the model considered in this paper has continuous sample paths, and it would be interesting to study the impact of the inclusion of jumps to its stochastic surplus process. 


\section{ACKNOWLEDGEMENTS}

The authors are grateful to the referees for useful comments that improved the presentation of the paper. Benjamin Avanzi acknowledges financial support from an Australian School of Business Special Research Grant. This research was also supported under Australian Research Council's Linkage Projects funding scheme (project number LP130100723). Vincent Tu acknowledges financial support from an Australian Postgraduate Award and supplementary scholarships provided by the Australian Business School, UNSW. The views expressed herein are those of the authors and are not necessarily those of the supporting organisations.

\section{REFERENCES}

Albrecher, H., Cheung, E.C.K. and Thonhauser, S. (2011a) Randomized observation periods for the compound poisson risk model: Dividends. Astin Bulletin, 41(2), 645-672.

Albrecher, H., Gerber, H.U. and SHIU, E.S.W. (2011b) The optimal dividend barrier in the gamma-omega model. European Actuarial Journal, 1(1), 43-55.

Albrecher, H. and ThONHAUSER, S. (2009) Optimality results for dividend problems in insurance. RACSAM Revista de la Real Academia de Ciencias; Serie A, Mathemáticas, 100(2), 295 320.

Asmussen, S. and Albrecher, H. (2010) Ruin Probabilities, 2nd Ed. Advanced Series on Statistical Science and Applied Probability, Vol. 14, Singapore: World Scientific.

AvAnZI, B. (2009) Strategies for dividend distribution: A review. North American Actuarial Journal, 13(2), 217-251.

Avanzi, B., Cheung, E.C.K., Wong, B. and Woo, J.-K. (January 2013) On a periodic dividend barrier strategy in the dual model with continuous monitoring of solvency. Insurance: Mathematics and Economics, 52(1), 98-113.

AvanzI, B., SHEN, J. and Wong, B. (2011) Optimal dividends and capital injections in the dual model with diffusion. ASTIN Bulletin, 41(2), 611-644.

AvANZI, B., TU, V. and WoNG, B. (2014) On optimal periodic dividend strategies in the dual model with diffusion. Insurance: Mathematics and Economics, 55, 210-224.

Bellman, R. (1954) The theory of dynamic programming. Technical report, DTIC Document.

BüHLManN, H. (1970) Mathematical Methods in Risk Theory. Grundlehren der mathematischen Wissenschaften. Berlin, Heidelberg, New York: Springer-Verlag.

Choi, M.C. and Cheung, E.C. (2014) On the expected discounted dividends in the cramérlundberg risk model with more frequent ruin monitoring than dividend decisions. Insurance: Mathematics and Economics, 59, 121-132.

DE FINETTI, B. (1957) Su un'impostazione alternativa della teoria collettiva del rischio. Transactions of the XVth International Congress of Actuaries, 2, 433-443.

GERBER, H.U. (1972) Games of economic survival with discrete- and continuous-income processes. Operations Research, 20(1), 37-45.

GERBER, H.U. (1974) The dilemma between dividends and safety and a generalization of the Lundberg-Cramér formulas. Scandinavian Actuarial Journal, 1974, 46-57.

Jagannathan, M., StePhens, C.P. and WeisbaCh, M.S. (September 2000). Financial flexibility and the choice between dividends and stock repurchases. Journal of Financial Economics, 57(3), 307-474.

JEANBLANC-PICQUÉ, M. and SHIRYAEV, A.N. (1995) Optimization of the flow of dividends. Russian Mathematical Surveys, 50(2), 257-277.

KLebANeR, F.C. (2005) Introduction to Stochastic Calculus with Applications, 2nd ed. London: Imperial College Press. 
Kyprianou, A.E. (2014) Introductory Lectures on Fluctuations of Levy Processes with Applications. 2nd edition. Springer-Verlag: Berlin.

MORILL, J.E. (1966) One-person games of economic survival. Naval Research Logistics Quarterly, 13(1), 49-69.

Morningstar (30 June 2014) 5 traps in using the dividend yield by Karl Siegling (last accessed on 18 December 2015 on http://www.morningstar.com.au/ funds/article/traps-dividend-yield/6574).

SHREVE, S.E., LEHOCZKY, J.P. and GAVER, D.P. (1984) Optimal consumption for general diffusions with absorbing and reflecting barriers. SIAM Journal on Control and Optimization, 22(1), 55 75.

WEI, J., WANG, R. and YANG, H. (2012) On the optimal dividend strategy in a regime-switching diffusion model. Advances in Applied Probability, 44(3), 886-906.

Westfarmers (20 August 2014) 2014 Capital Management Initiative - A quick guide (last accessed on 18 December 2015 on http://www.wesfarmers.com.au/investors/ shareholder-information/capital-returns-rights-issues -and-rearrangements).

Woodside Petroleum (23 April 2013) Special dividends and dividend payout announcement (last accessed on 18 December 2015 on http://www.woodside.com.au/investorsmedia/shareholders-services/pages/dividend-information.aspx).

ZhANG, Z. and Cheung, E.C.K. (2014) The Markov additive risk process under an Erlangized dividend barrier strategy. Methodology and Computing in Applied Probability. DOI: 10.1007/s11009-014-9414-7.

BENJAMIN AVANZI

School of Risk and Actuarial Studies

UNSW Australia Business School

UNSW Sydney NSW 2052, Australia

Département de Mathématiques et de Statistique

Université de Montréal

Montréal QC H3T 1J4, Canada

E-Mail:b.avanzi@unsw.edu.au

VINCENT TU (Corresponding author)

School of Risk and Actuarial Studies

UNSW Australia Business School

UNSW Sydney NSW 2052, Australia

E-Mail:v.tu@unsw.edu.au

BERNARD WONG

School of Risk and Actuarial Studies

UNSW Australia Business School

UNSW Sydney NSW 2052, Australia

E-Mail: bernard.wong@unsw.edu.au 


\section{APPENDIX}

\section{A.1. Existence and uniqueness of $b_{c}^{*}-b_{p}^{*}$}

From (3.23), $b_{c}^{*}-b_{p}^{*}$ is the root of the following function $h(\xi)$,

$$
h(\xi)=\left(\eta_{c}-\eta_{p} \frac{\gamma}{\gamma+\delta}\right)\left(\frac{s_{\gamma}}{s_{\gamma}-r_{\gamma}} e^{-r_{\gamma} \xi}+\frac{r_{\gamma}}{r_{\gamma}-s_{\gamma}} e^{-s_{\gamma} \xi}\right)-\eta_{p} \frac{\delta}{\gamma+\delta}, \quad x \in(0, \infty) .
$$

First, note that the function $h(\xi)$ has a $y$-intercept,

$$
h(0)=\left(\eta_{c}-\eta_{p} \frac{\gamma}{\gamma+\delta}\right)\left(\frac{s_{\gamma}}{s_{\gamma}-r_{\gamma}}+\frac{r_{\gamma}}{r_{\gamma}-s_{\gamma}}\right)-\eta_{p} \frac{\delta}{\gamma+\delta}=\eta_{c}-\eta_{p},
$$

which is strictly negative as long as $\eta_{c}<\eta_{p}$. Next, we obtain the first derivative of $h(\xi)$,

$$
h^{\prime}(\xi)=-\left(\eta_{c}-\eta_{p} \frac{\gamma}{\gamma+\delta}\right) \frac{r_{\gamma} s_{\gamma}}{s_{\gamma}-r_{\gamma}}\left(e^{-r_{\gamma} \xi}-e^{-s_{\gamma} \xi}\right) .
$$

Since $h(\xi)$ is defined on positive real numbers and using the signs of $r_{\gamma}$ and $s_{\gamma}$, we have $e^{-r_{\gamma} \xi}-e^{-s_{\gamma} \xi}<0$ for all $\xi \in(0, \infty), r_{\gamma} s_{\gamma}<0$ and $s_{\gamma}-r_{\gamma}<0$. Combining these facts, the sign of $h^{\prime}(\xi)$ is controlled by the sign of $\eta_{c}-\eta_{p} \gamma /(\gamma+\delta)$. Since $h(\xi)$ is continuous and has a negative $y$-intercept, $h(\xi)$ has a unique positive root if and only if $h$ is strictly increasing, i.e.,

$$
\begin{aligned}
& \eta_{c}-\eta_{p} \frac{\gamma}{\gamma+\delta} \leq 0 \Longleftrightarrow h^{\prime}(\xi) \leq 0 \Longleftrightarrow h(\xi) \text { has no root; } \\
& \eta_{c}-\eta_{p} \frac{\gamma}{\gamma+\delta}>0 \Longleftrightarrow h^{\prime}(\xi)>0 \Longleftrightarrow h(\xi) \text { has a unique positive root. }
\end{aligned}
$$

\section{A.2. $G_{H}\left(x ; b_{p}^{*}, b_{c}^{*}\right)$ is an increasing and concave function}

First, we use the explicit form of $G_{L}(x)$ in (3.32) and obtain its first and second derivatives,

$$
G_{L}^{\prime}(x)=\eta_{p} \frac{r_{0} e^{r_{0} x}-s_{0} e^{s_{0} x}}{r_{0} e^{r_{0} b_{p}^{*}}-s_{0} e^{s_{0} b_{p}^{*}}} \quad \text { and } \quad G_{L}^{\prime \prime}(x)=\eta_{p} \frac{r_{0}^{2} e^{r_{0} x}-s_{0}^{2} e^{s_{0} x}}{r_{0} e^{r_{0} b_{p}^{*}}-s_{0} e^{s_{0} b_{p}^{*}}}, \quad x \in\left[0, b_{p}^{*}\right) .
$$

We first show that $G_{L}^{\prime \prime}(x)<0$ for $x \in\left[0, b_{p}^{*}\right)$. Combining the explicit form of $G_{M}\left(b_{p}^{*}\right)$ in (3.28) and equation (3.23), we have an alternative form of $G_{M}\left(b_{p}^{*}\right)$ after some algebra

$$
G_{M}\left(b_{p}^{*}\right)=\eta_{p}\left(\frac{\mu}{\delta}-\frac{e^{r_{\gamma}\left(b_{c}^{*}-b_{p}^{*}\right)}-e^{s_{\gamma}\left(b_{c}^{*}-b_{p}^{*}\right)}}{r_{\gamma} e^{r_{\gamma}\left(b_{c}^{*}-b_{p}^{*}\right)}-s_{\gamma} e^{s_{\gamma}\left(b_{c}^{*}-b_{p}^{*}\right)}}\right) .
$$


From (A.7), we see that $G_{M}\left(b_{p}^{*}\right)<\eta_{p} \mu / \delta$ and we can establish the following inequality,

$$
G_{M}\left(b_{p}^{*}\right)<\eta_{p} \frac{\mu}{\delta} \Longleftrightarrow G_{M}\left(b_{p}^{*}\right)<\eta_{p} \frac{s_{0}+r_{0}}{s_{0} r_{0}},
$$

which further becomes

$$
\begin{gathered}
G_{M}\left(b_{p}^{*}\right)<\eta_{p} \frac{s_{0}+r_{0}}{s_{0} r_{0}} \Longleftrightarrow \frac{\eta_{p}-s_{0} G_{M}\left(b_{p}^{*}\right)}{\eta_{p}-r_{0} G_{M}\left(b_{p}^{*}\right)}<\frac{s_{0}^{2}}{r_{0}^{2}} \\
\Longleftrightarrow b_{p}^{*}<\frac{1}{r_{0}-s_{0}} \log \left(\frac{s_{0}^{2}}{r_{0}^{2}}\right) \Longleftrightarrow r_{0}^{2} e^{r_{0} b_{p}^{*}}-s_{0}^{2} e^{s_{0} b_{p}^{*}}<0 .
\end{gathered}
$$

Since $r_{0}^{2} e^{r_{0} x}-s_{0}^{2} e^{s_{0} x}$ is an increasing function of $x$, we must have $r_{0}^{2} e^{r_{0} x}-s_{0}^{2} e^{s_{0} x}<$ 0 and $G_{L}^{\prime \prime}(x)<0$ for $x \in\left[0, b_{p}^{*}\right)$. This shows that $G_{L}^{\prime}(x)$ is a decreasing function and we have

$$
\eta_{p}=G_{L}^{\prime}\left(b_{p}^{*}\right)<G_{L}^{\prime}(x) \leq G_{L}^{\prime}(0) \quad x \in\left[0, b_{p}^{*}\right),
$$

which shows that $G_{L}^{\prime}(x)>0$ for $x \in\left[0, b_{p}^{*}\right)$. This completes the proof that $G_{L}(x)$ is an increasing and concave function on $x \in\left[0, b_{p}^{*}\right)$.

Next, we use the explicit form of $G_{M}(x)$ in (3.22) and compute its first and second derivatives

$$
\begin{aligned}
& G_{M}^{\prime}(x)=\left(\eta_{c}-\eta_{p} \frac{\gamma}{\gamma+\delta}\right)\left[\frac{s_{\gamma}}{s_{\gamma}-r_{\gamma}} e^{r_{\gamma}\left(x-b_{c}^{*}\right)}+\frac{r_{\gamma}}{r_{\gamma}-s_{\gamma}} e^{s_{\gamma}\left(x-b_{c}^{*}\right)}\right]+\eta_{p} \frac{\gamma}{\gamma+\delta}, \\
& G_{M}^{\prime \prime}(x)=\left(\eta_{c}-\eta_{p} \frac{\gamma}{\gamma+\delta}\right) \frac{s_{\gamma} r_{\gamma}}{s_{\gamma}-r_{\gamma}}\left(e^{r_{\gamma}\left(x-b_{c}^{*}\right)}-e^{s_{\gamma}\left(x-b_{c}^{*}\right)}\right) .
\end{aligned}
$$

Using the signs of $r_{\gamma}>0$ and $s_{\gamma}<0$, we have $G_{M}^{\prime \prime}(x)<0$. Next, since $G_{M}^{\prime \prime}(x)$ is concave, so $G_{M}^{\prime}(x)$ is a decreasing function and we must have

$$
\eta_{c}=G_{M}^{\prime}\left(b_{c}^{*}\right)<G_{M}^{\prime}(x) \quad x \in\left[b_{p}^{*}, b_{c}^{*}\right),
$$

which shows that $G_{M}^{\prime}(x)>0$ for $x \in\left[b_{p}^{*}, b_{c}^{*}\right)$. This completes the proof that $G_{M}(x)$ is an increasing and concave function on $x \in\left[b_{p}^{*}, b_{c}^{*}\right.$ ).

By construction, $G_{U}(x)$ in (3.7) is a linear function with positive first derivative. So $G_{U}(x)$ is an increasing and concave function on $x \in\left[b_{c}^{*}, \infty\right)$. 


\section{A.3. Existence and uniqueness of $b_{c}^{*}$ in a liquidation-at-first-periodic-opportunity strategy}

In an optimal hybrid barrier strategy with liquidation-at-first-periodicopportunity, we assume that proportional transaction costs satisfy

$$
\eta_{c}-\eta_{p} \frac{\gamma}{\gamma+\delta}>0, \text { and that the drift } \mu>0 .
$$

Then, from (3.42) $b_{c}^{*}$ is the solution to the following equation $g(\xi)$ :

$$
g(\xi)=\left(\eta_{c}-\eta_{p} \frac{\gamma}{\gamma+\delta}\right)\left(\frac{s_{\gamma}}{s_{\gamma}-r_{\gamma}} \frac{e^{-r_{\gamma} \xi}}{r_{\gamma}}+\frac{r_{\gamma}}{r_{\gamma}-s_{\gamma}} \frac{e^{-s_{\gamma} \xi}}{s_{\gamma}}\right)+\eta_{p} \frac{\gamma}{\gamma+\delta} \frac{\mu}{\gamma+\delta} .
$$

The derivative of $g(\xi)$ is

$$
g^{\prime}(\xi)=-\left(\eta_{c}-\eta_{p} \frac{\gamma}{\gamma+\delta}\right)\left(\frac{s_{\gamma}}{s_{\gamma}-r_{\gamma}} e^{-r_{\gamma} \xi}+\frac{r_{\gamma}}{r_{\gamma}-s_{\gamma}} e^{-s_{\gamma} \xi}\right) .
$$

Since we have $\eta_{c}-\eta_{p} \frac{\gamma}{\gamma+\delta}>0$ and using the signs of $r_{\gamma}$ and $s_{\gamma}$, the derivative $g^{\prime}(\xi)$ is strictly negative. Furthermore, as $\xi \rightarrow \infty$, we have

$$
\begin{aligned}
\lim _{\xi \rightarrow \infty} g(\xi)= & \left(\eta_{c}-\eta_{p} \frac{\gamma}{\gamma+\delta}\right)\left(\frac{s_{\gamma}}{s_{\gamma}-r_{\gamma}} \frac{e^{-r_{\gamma} \xi}}{r_{\gamma}}+\frac{r_{\gamma}}{r_{\gamma}-s_{\gamma}} \frac{e^{-s_{\gamma} \xi}}{s_{\gamma}}\right) \\
& +\eta_{p} \frac{\gamma}{\gamma+\delta} \frac{\mu}{\gamma+\delta}=-\infty .
\end{aligned}
$$

Therefore, a continuous function $g(\xi)$ has a unique positive root if and only if $g(0)>0$, which can be verified

$$
\begin{aligned}
g(0) & =\left(\eta_{c}-\eta_{p} \frac{\gamma}{\gamma+\delta}\right)\left(\frac{s_{\gamma}}{s_{\gamma}-r_{\gamma}} \frac{1}{r_{\gamma}}+\frac{r_{\gamma}}{r_{\gamma}-s_{\gamma}} \frac{1}{s_{\gamma}}\right)+\eta_{p} \frac{\gamma}{\gamma+\delta} \frac{\mu}{\gamma+\delta} \\
& =\eta_{c} \frac{\mu}{\gamma+\delta}>0 .
\end{aligned}
$$

\section{A.4. $G_{H}\left(x ; 0, b_{c}^{*}\right)$ is an increasing and concave function}

Using the explicit form of $F_{L}(x)$ in derived in (3.41), we can obtain its first and second derivatives. For $x \in\left[0, b_{c}^{*}\right)$

$$
\begin{aligned}
& F_{L}^{\prime}(x)=\left(\eta_{c}-\eta_{p} \frac{\gamma}{\gamma+\delta}\right)\left(\frac{s_{\gamma}}{s_{\gamma}-r_{\gamma}} e^{r_{\gamma}\left(x-b_{c}^{*}\right)}+\frac{r_{\gamma}}{r_{\gamma}-s_{\gamma}} e^{s_{\gamma}\left(x-b_{c}^{*}\right)}\right)+\frac{\eta_{p} \gamma}{\gamma+\delta}>0, \\
& F_{L}^{\prime \prime}(x)=\left(\eta_{c}-\eta_{p} \frac{\gamma}{\gamma+\delta}\right) \frac{s_{\gamma} r_{\gamma}}{s_{\gamma}-r_{\gamma}}\left(e^{r_{\gamma}\left(x-b_{c}^{*}\right)}-e^{s_{\gamma}\left(x-b_{c}^{*}\right)}\right)<0 .
\end{aligned}
$$


So $F_{L}(x)$ is an increasing and concave function on $x \in\left[0, b_{c}^{*}\right)$ and $F_{U}(x)$ is by-construction linear on $\left[b_{c}^{*}, \infty\right)$.

\section{A.5. Proof of Lemma 4.2}

Proof 2. First, the underlying strategies of the optimal hybrid barrier type, therefore the proportional costs satisfy $\eta_{c}>\eta_{p} \gamma / \gamma+\delta$ and $r_{\gamma}>0$ and $s_{\gamma}<0$ are the roots of the equation (3.12) with $\delta=\delta+\gamma$. Before we begin the proof, let us re-state the insufficient-prospect condition. Let $b>0$ be the solution of the equation $h(\xi)$ in (3.24). Then, the inequality for the insufficient-prospect condition is

$$
\begin{aligned}
G(b) & =\eta_{p} \frac{\mu}{\delta} \frac{\gamma}{\gamma+\delta}+\frac{\gamma+\delta}{\delta}\left(\eta_{c}-\eta_{p} \frac{\gamma}{\gamma+\delta}\right)\left(\frac{s_{\gamma}}{s_{\gamma}-r_{\gamma}} \frac{e^{-r_{\gamma} b}}{r_{\gamma}}+\frac{r_{\gamma}}{r_{\gamma}-s_{\gamma}} \frac{e^{-s_{\gamma} b}}{s_{\gamma}}\right) \\
& \leq 0 .
\end{aligned}
$$

Our goal is to show that $G(b) \leq 0 \Longleftrightarrow F_{L}^{\prime}(0) \leq \eta_{p}$. Recall from (3.41), we have

$$
F_{L}^{\prime}(0)=\left(\eta_{c}-\eta_{p} \frac{\gamma}{\gamma+\delta}\right)\left(\frac{s_{\gamma}}{s_{\gamma}-r_{\gamma}} e^{-r_{\gamma} b_{c}^{*}}+\frac{r_{\gamma}}{r_{\gamma}-s_{\gamma}} e^{-s_{\gamma} b_{c}^{*}}\right)+\eta_{p} \frac{\gamma}{\gamma+\delta},
$$

where $b_{c}^{*}$ is the solution to the equation

$$
\left(\eta_{c}-\eta_{p} \frac{\gamma}{\gamma+\delta}\right)\left(\frac{s_{\gamma}}{s_{\gamma}-r_{\gamma}} \frac{e^{-r_{\gamma} b_{c}^{*}}}{r_{\gamma}}+\frac{r_{\gamma}}{r_{\gamma}-s_{\gamma}} \frac{e^{-s_{\gamma} b_{c}^{*}}}{s_{\gamma}}\right)+\frac{\eta_{p} \gamma}{\gamma+\delta} \frac{\mu}{\gamma+\delta}=0 .
$$

Note that (A.23) achieves the equality of (A.21), (i.e., $G(b) \leq G\left(b_{c}^{*}\right)=0$ ). Next, we can show that $G(b)$ is a decreasing function of $b$,

$$
\frac{d}{d b} G(b)=-\frac{\gamma+\delta}{\delta}\left(\eta_{c}-\eta_{p} \frac{\gamma}{\gamma+\delta}\right)\left(\frac{s_{\gamma}}{s_{\gamma}-r_{\gamma}} e^{-r_{\gamma} b}+\frac{r_{\gamma}}{r_{\gamma}-s_{\gamma}} e^{-s_{\gamma} b}\right)<0 .
$$

Therefore, we have $G(b) \leq G\left(b_{c}^{*}\right) \Longleftrightarrow b_{c}^{*} \leq b$. Next, since $b$ is the solution to $h(b)=0$ and $h$ is a strictly increasing function (see Section A.1),

$$
\begin{aligned}
G(b) & \leq G\left(b_{c}^{*}\right)=0 \\
& \Longleftrightarrow b_{c}^{*} \leq b \\
& \Longleftrightarrow h\left(b_{c}^{*}\right) \leq h(b)=0 \\
& \Longleftrightarrow\left(\eta_{c}-\eta_{p} \frac{\gamma}{\gamma+\delta}\right)\left(\frac{s_{\gamma}}{s_{\gamma}-r_{\gamma}} e^{-r_{\gamma} b_{c}^{*}}+\frac{r_{\gamma}}{r_{\gamma}-s_{\gamma}} e^{-s_{\gamma} b_{c}^{*}}\right) \leq \eta_{p} \frac{\delta}{\gamma+\delta} \\
& \Longleftrightarrow F^{\prime}(0) \leq \eta_{p} .
\end{aligned}
$$

Since all arguments are reversible and the proof is complete. 